AIAA-2003-1246

\title{
Simulations of 6-DOF Motion with a Cartesian Method
}

\author{
Scott M. Murman* \\ ELORET \\ Moffett Field, CA 94035 \\ smurman@nas.nasa.gov \\ Michael J. Aftosmis ${ }^{\dagger}$ \\ NASA Ames Research Center
}

Marsha J. Berger ${ }^{\ddagger}$

Courant Institute

251 Mercer St.

New York, NY 10012

\begin{abstract}
Coupled 6-DOF/CFD trajectory predictions using an automated Cartesian method are demonstrated by simulating a GBU-32/JDAM store separating from an F-18C aircraft. Numerical simulations are performed at two Mach numbers near the sonic speed, and compared with flight-test telemetry and photographic-derived data. Simulation results obtained with a sequentialstatic series of flow solutions are contrasted with results using a time-dependent flow solver. Both numerical methods show good agreement with the flight-test data through the first half of the simulations. The sequential-static and timedependent methods diverge over the last half of the trajectory prediction, after the store produces peak angular rates. A cost comparison for the Cartesian method is inchuded, in terms of absolute cost and relative to computing uncoupled 6-DOF trajectories. A detailed description of the 6-DOF method, as well as a verification of its accuracy, is provided in an appendix.
\end{abstract}

*Member AIAA

${ }^{\dagger}$ Senior Member AIAA

$\ddagger$ Member AIAA

Copyright (C)2003 by the American Institute of Aeronautics and Astronautics, Inc. No copyright is asserted in the United States under Title 17, U. S. Code. The U. S. Government has a royalty-free license to exercise all rights under the copyright claimed herein for Governmental purposes. All other rights are reserved by the copyright owner. 


\section{Introduction}

Trajectory prediction is an important element in Computational Fluid Dynamics (CFD) simulations of bodies undergoing unconstrained, or partially constrained motion. Modeling this behavior involves integrating the Newton-Euler equations for six-degree-of-freedom (6-DOF) rigid-body motion, in response to aerodynamic and other externally applied loads. Numerous important applications for such models exist, including store separation from an aircraft, booster separation from a space launch vehicle, canopy or shroud separation, and simulation of flight control systems. Many CFD technologies have been demonstrated for 6-DOF simulations, including structured overset $[1,2]$, unstructured tetrahedral [3, 4], and hybrid prismatic/Cartesian[5]. The current work demonstrates an integrated package for performing 6-DOF simulations couple with an inviscid, Cartesian embedded-boundary method.

Such non-body-fitted, Cartesian methods are particularly interesting for 6-DOF applications since they can be made both extremely fast and robust, and the volume meshing can proceed automatically. Moreover, they are comparatively insensitive to the complexity of the input geometry since the surface description is decoupled from the volume mesh. In the current work, the "cut-cell" Cartesian meshing scheme of Aftosmis et al.[6] is utilized. The intersection of the solid geometry with the regular Cartesian hexahedra is computed, and polyhedral cells are formed which contain the embedded boundary. This volume meshing procedure is robust, computationally efficient, and does not require user intervention.

In order to demonstrate the utility of the Cartesian 6-DOF package, a U.S. Navy GBU-32 Joint Direct Attack Munition (JDAM) store (cf. Fig. 1) separating from an $\mathrm{F} / \mathrm{A}-18 \mathrm{C}$ is simulated using both sequential-static and time-dependent methods. This transonic JDAM separation was put forward by the Navy as a "challenge" to the CFD community because it exhibited behavior that could not reliably be predicted with conventional store separation analysis tools (cf. Cenko $[7,8]$ ). The JDAM separation provides an attractive demonstration case because it contains a complex aircraft geometry, flight telemetry and photographic-derived quantitative data, and also because it has been simulated by numerous other CFD methods[9-15]. These previous CFD simulations can be broken into two broad classes; those which computed a set of static solutions which were used with a store trajectory simulation package, and those which computed the trajectory of the store within the CFD simulation process. Both of these approaches are supported with the current methods and a cost comparison will be presented.

The discussion begins by reviewing the geometry used in the simulations, and briefly outlines the numerical scheme. Next it presents computed results for the JDAM separation flight conditions just below and just above sonic speed $\left(M_{\infty}=\right.$ 0.962 and 1.055). These results are directly compared to both fight telemetry and photographic-derived data. The computational cost for the current method is provided, along with a summary of the current results and topics for future work. A detailed description and verification of the stand-alone 6-DOF package used with the 

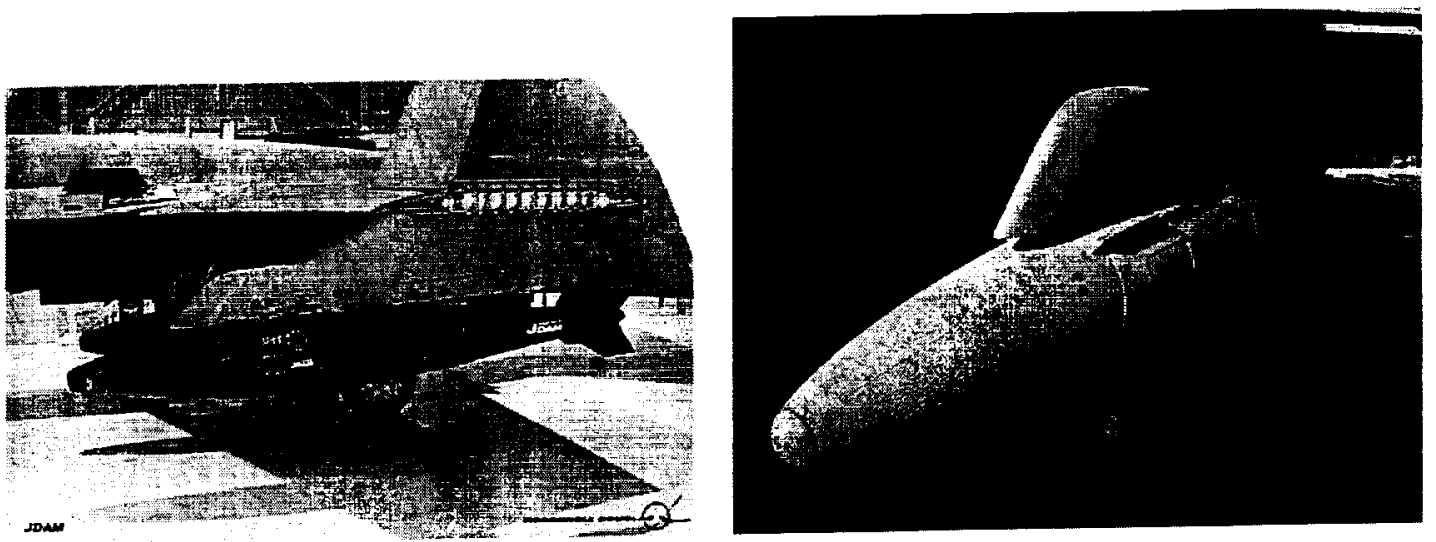

Figure 1: L.S. Navy GBU-32 Joint Direct Attack Munition (JDAM) on the F/A-18C wing pylon. The dark green fins and center carriage provide the JDAM GPS guidance augmentation system which can be retrofit on a general purpose unit such as (in this case) an Mk-84.

current scheme is included in an appendix.

\section{Numerical Scheme}

\subsection{Geometry and Computational Mesh}

The surface geometry was provided as a set of structured surface patches. These were converted to water-tight surface triangulations of the various components. The addition of an internal duct connecting the engine diffuser face to the exit nozzle was required in order to form a water-tight fuselage. The component geometry for the complete $\mathrm{F} / \mathrm{A}-18 \mathrm{C}$ is shown in Fig. 2, with water-tight components shown with different colors. All of the major components of the geometry are modeled, including the empennage, AIM- 7 wingtip missile and rail, wing with leading-edge extensions (LEX), the LEX fence, the engine inlet including boundary layer vents, and the wing pylons holding a 330 gal. external fuel tank (EFT) inboard, and the GBU-32 JDAM outboard. Note that the flight configuration did not contain the AIM-7 wingtip missiles. Fig. 3 shows a closeup view of the JDAM in its initial position beneath the port, outboard wing pylon. The attachment hardware and ejector mechanism is not modeled.

Using the automated Cartesian meshing scheme of Aftosmis et al.[6], the triangulated surface was used to generate an unstructured Cartesian volume mesh by subdividing the computational domain based upon the geometry. The sharp geometric features contain refined cells, while areas away from the geometry maintain a relatively coarse spacing. The intersection of the solid geometry with the the regular Cartesian hexahedra is computed, and polyhedral cells are formed which contain the embedded boundary. Regions interior to the solid geometry are removed. The solidwall boundary conditions for the flow solver are then specified within these cut-cell 


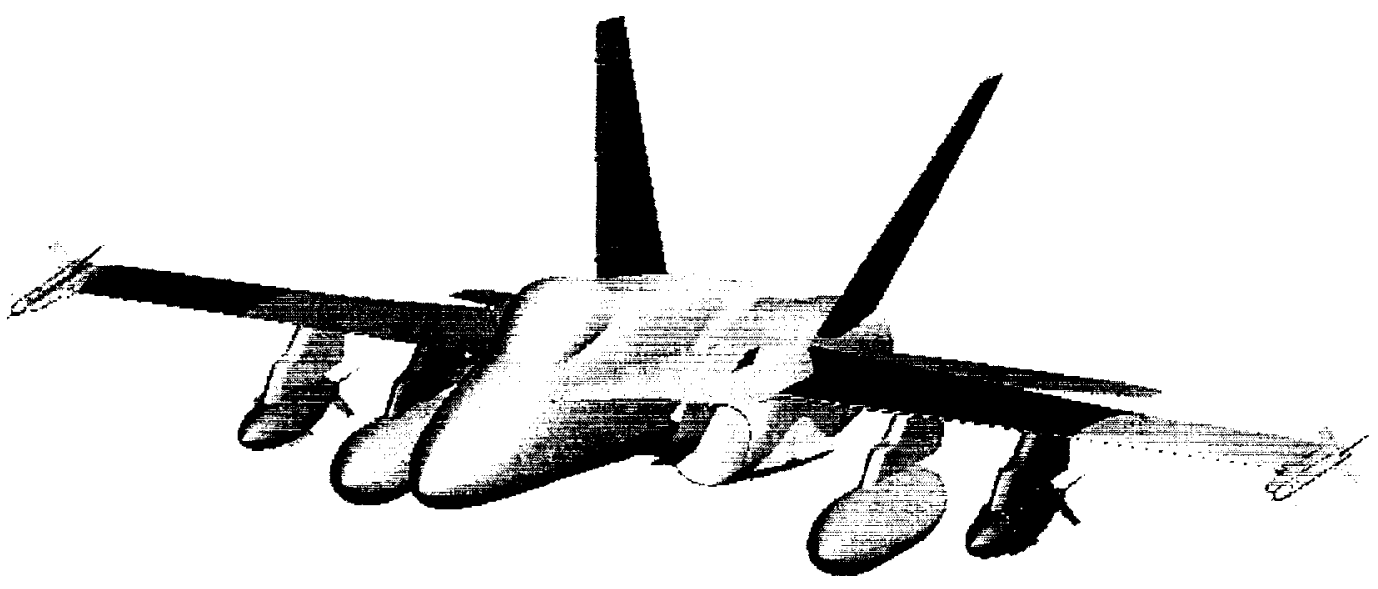

Figure 2: F/A-18C surface geometry. Water-tight components are shown with different colors.

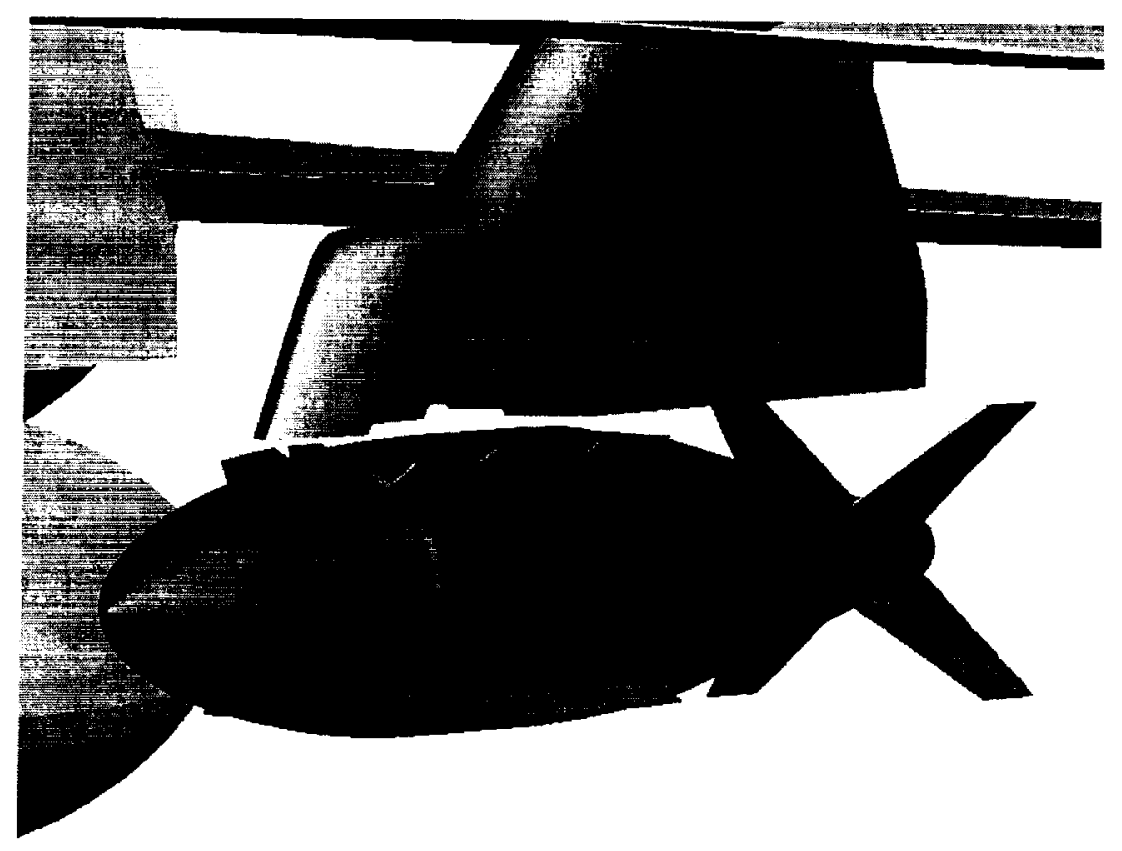

Figure 3: Closeup view of triangulated GBU-32/JDAM in its initial position beneath the wing pylon. 

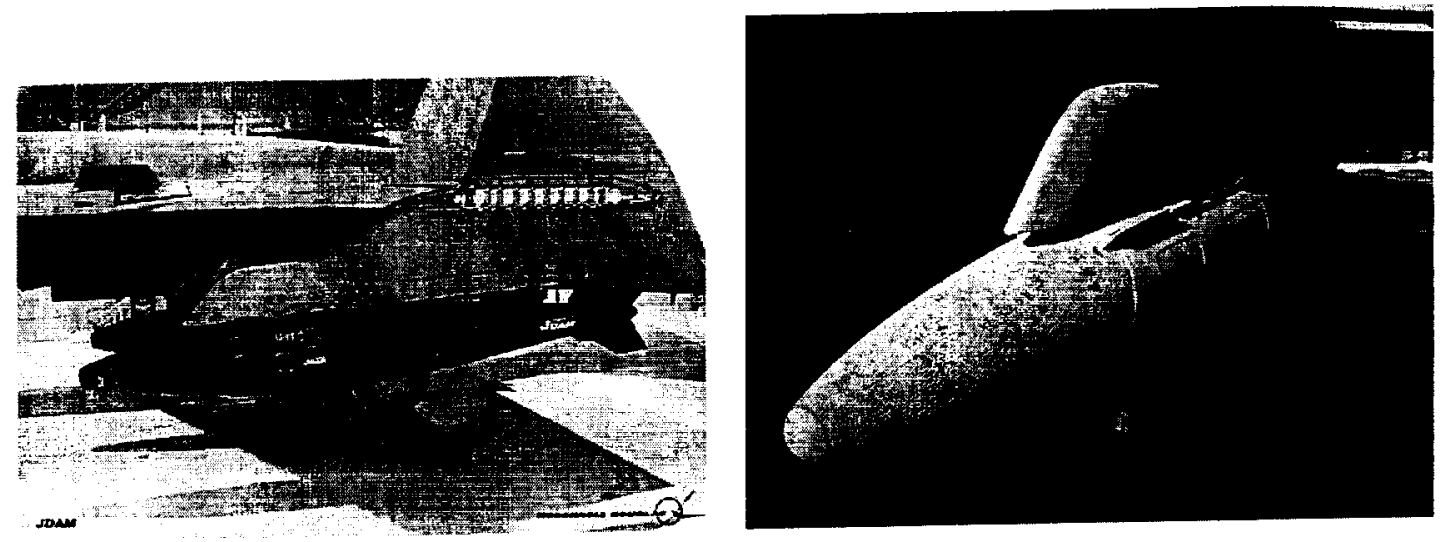

Figure 1: U.S. Navy GBU-32 Joint Direct Attack Munition (JDAM) on the F/A-18C wing pylon. The dark green fins and center carriage provide the JDAM GPS guidance augmentation system which can be retrofit on a general purpose unit such as (in this case) an Mk-84.

current scheme is included in an appendix.

\section{Numerical Scheme}

\subsection{Geometry and Computational Mesh}

The surface geometry was provided as a set of structured surface patches. These were converted to water-tight surface triangulations of the various components. The addition of an internal duct connecting the engine diffuser face to the exit nozzle was required in order to form a water-tight fuselage. The component geometry for the complete $\mathrm{F} / \mathrm{A}-18 \mathrm{C}$ is shown in Fig. 2, with water-tight components shown with different colors. All of the major components of the geometry are modeled, including the empennage, AIM- 7 wingtip missile and rail, wing with leading-edge extensions (LEX), the LEX fence, the engine inlet including boundary layer vents, and the wing pylons holding a 330 gal. external fuel tank (EFT) inboard, and the GBU-32 JDAM outboard. Note that the flight configuration did not contain the AIM-7 wingtip missiles. Fig. 3 shows a closeup view of the JDAM in its initial position beneath the port, outboard wing pylon. The attachment hardware and ejector mechanism is not modeled.

Using the automated Cartesian meshing scheme of Aftosmis et al.[6], the triangulated surface was used to generate an unstructured Cartesian volume mesh by subdividing the computational domain based upon the geometry. The sharp geometric features contain refined cells, while areas away from the geometry maintain a relatively coarse spacing. The intersection of the solid geometry with the the regular Cartesian hexahedra is computed, and polyhedral cells are formed which contain the embedded boundary. Regions interior to the solid geometry are removed. The solidwall boundary conditions for the flow solver are then specified within these cut-cell 


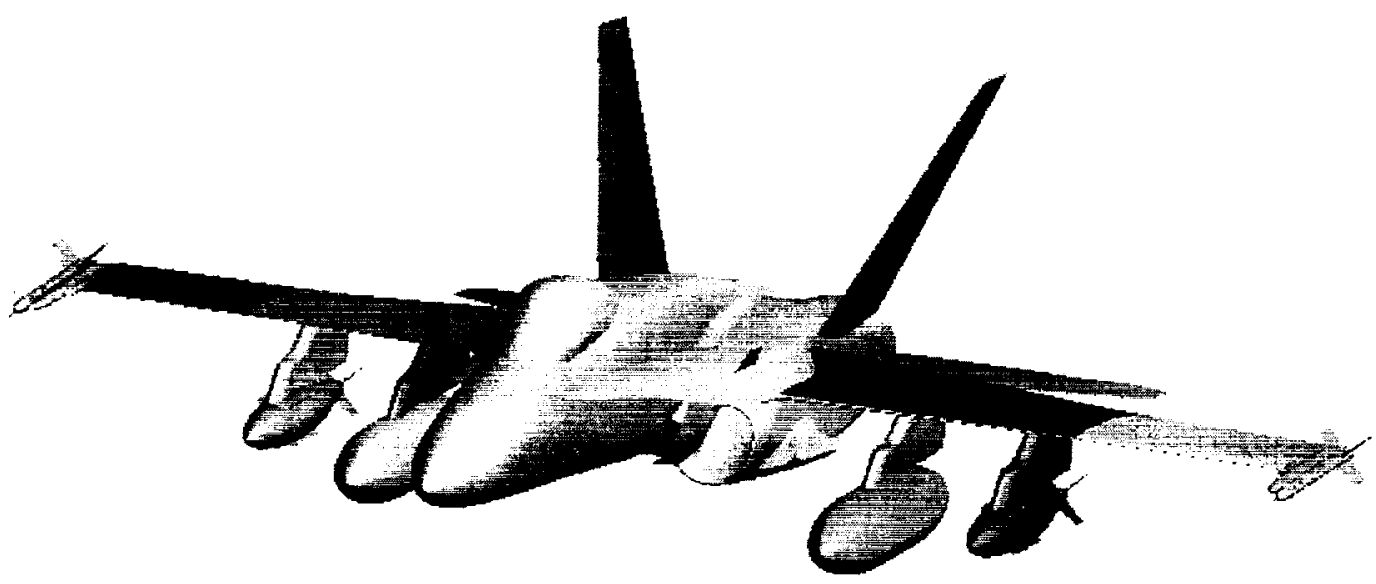

Figure 2: F/A-18C surface geometry. Water-tight components are shown with different colors.

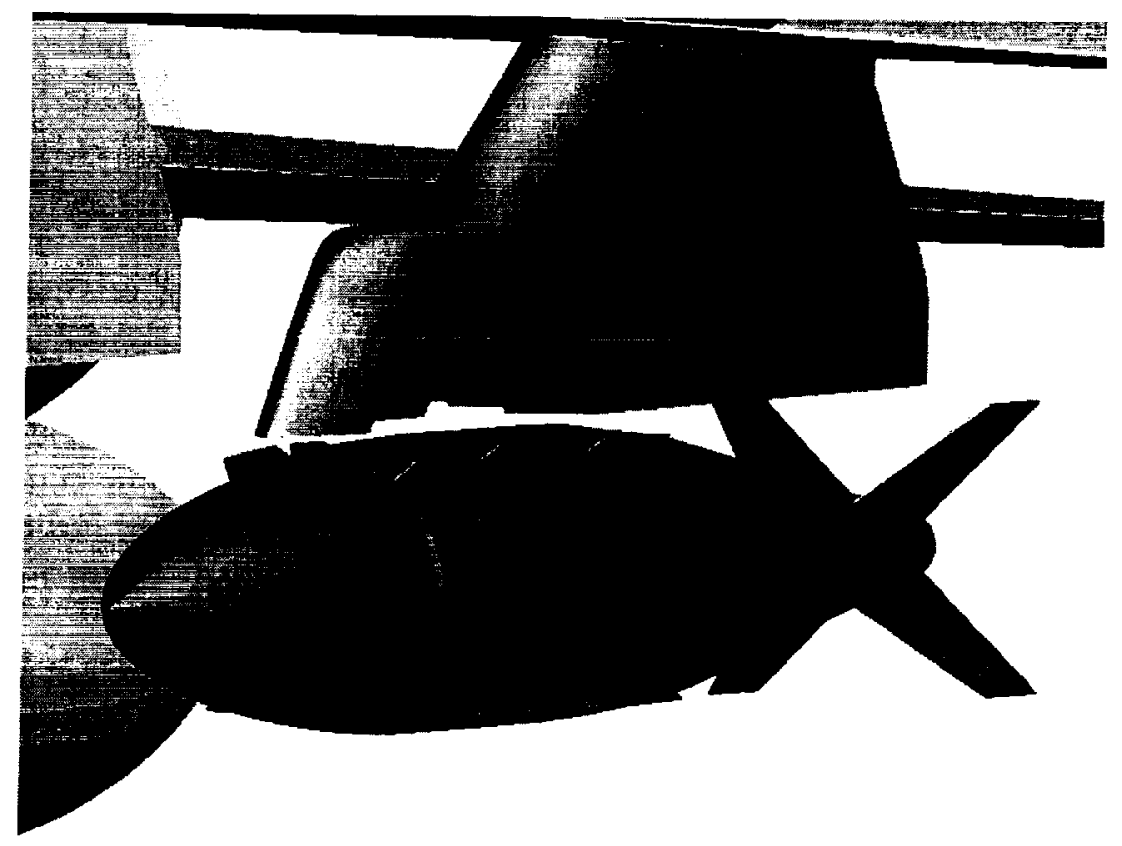

Figure 3: Closeup view of triangulated GBU-32/JDAM in its initial position beneath the wing pylon. 


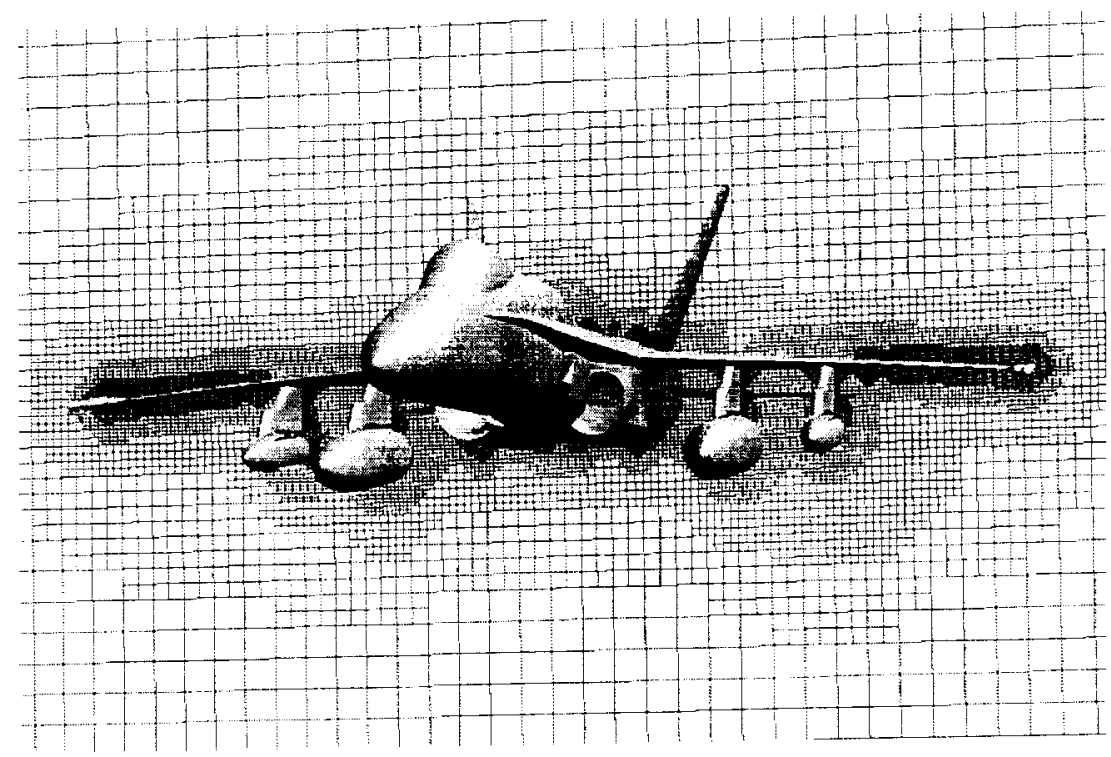

Figure 4: Cutting plane through the Cartesian volume mesh.

polyhedra.

In addition to mesh refinement near geometric features, pre-specified adaption regions are arranged around the major components of the F-18 aircraft to resolve the shock structures that occur at the current flow conditions. The adaption region which surrounds the JDAM translates with the center of mass (c.m.) location as the store drops. In the future, these pre-specified regions will be replaced with automated solution and geometry adaptation similar to the steady-state scheme outlined by Aftosmis and Berger[16]. A mesh refinement comparison was performed for the static, steady-state simulation with the JDAM in its initial position at the Mach 0.962 flight conditions (cf. Table 2). The resulting volume mesh is isotropic and contains $3.8 \mathrm{M}$ cells with a surface resolution of $1.0 \mathrm{in}$. A volume mesh cutting plane through the wing is shown in Fig. 4. Details of the mesh adaptation to the moving geometry will be presented in Sec. 3 .

\subsection{Flow Solver}

The inviscid, parallel multigrid flow solver of Aftosmis et al.[17] provides static, steady-state flow simulations for Cartesian meshes. Recently, this flow solver has been extended to provide capability for time-dependent flows, including dynamic simulations with rigid bodies in relative motion $[18,19]$. The current work implements an independent 6-DOF module which can be utilized as a stand-alone external application, or tightly coupled within the time-dependent flow solver. A flow diagram for the 6-DOF/CFD simulation process is shown in Fig. 5. The XML4CFD interface[20] is utilized to integrate the independent mesh generation, flow solver, post-processing, and 6-DOF steps into a unified computational framework. 


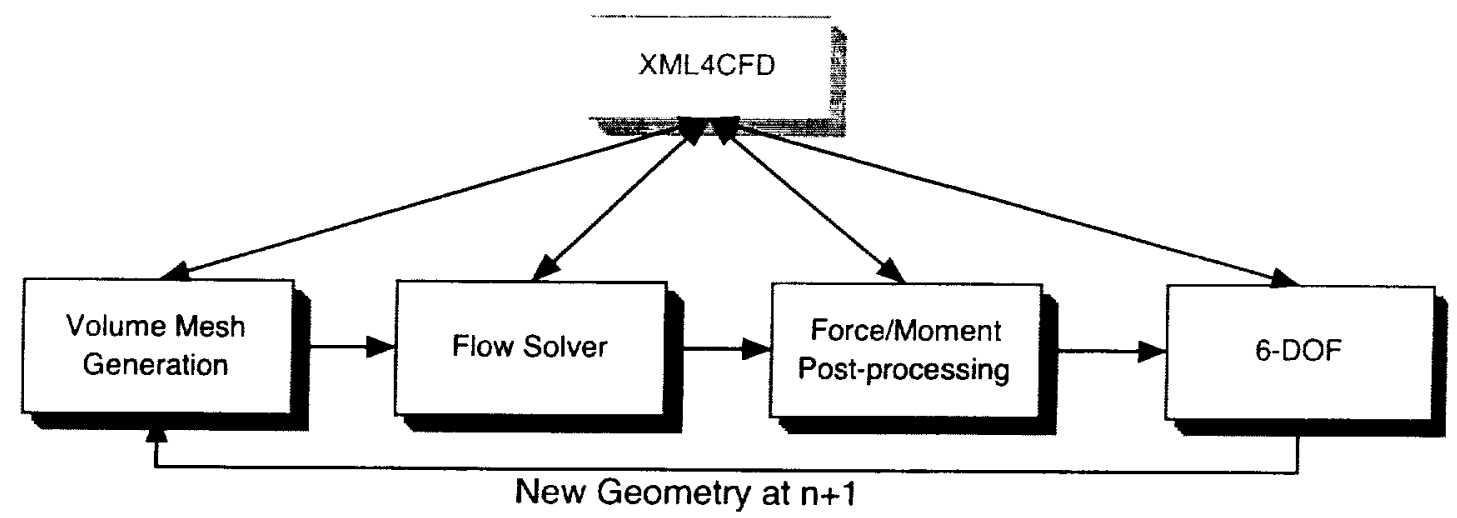

Figure 5: Process diagram for 6-DOF simulations. The red-colored processes are serial and the blue parallel. The XML4CFD interface[20] provides a single repository and API for the moving-body information required by the separate processes.

The 6-DOF module decomposes the rigid-body motion into a translation of the center of mass and a rotation about an axis passing through the c.m. location. The position of the c.m. is updated using Newton's laws of motion in the inertial frame, while the rotation of the body is determined by numerically integrating Euler's equations of motion in a body principal-axis system. The rotational position of the body is specified using Euler parameters, which are updated by numerical integration of the angular velocity. General external applied forces, in either the aerodynamic or body coordinate frames, can be specified. A detailed discussion of the 6-DOF model, along with validation test cases is presented in App. A.

\subsection{Ejector Force Model}

The JDAM is forced away from its wing pylon by means of identical piston ejectors located in the lateral plane of the store, $-10.11 \mathrm{in}$. forward of the c.m., and 9.89 in. aft. The ejectors extend during operation for 6 in., and the force of each ejector is a polynomial function of this stroke extension (cf. Cenko[7]). As the store moves away from the pylon it begins to pitch and yaw due to aerodynamic forces, and the stroke length of the individual ejectors responds asymmetrically. This response of the ejectors to the store motion is modeled, and the result is presented as a function of time for each piston. This modeling process for the F-18/JDAM is described by Fortin et al.[14], and the results are presented in Table 1. A consistent theme with previous simulations is that this ejector model is limited (cf. $[8,9,14,15]$ ). Since even slight errors in the initial trajectory of the store can become augmented as the separation simulation is marched forward in time, researchers have modified either the ejector model, or the computed JDAM trajectory, in order to provide a realistic store separation. Physically, the JDAM is constrained by the ejector mechanism, which is not accounted for in simplistic models. For example, the JDAM cannot be allowed to pitch nose-down without bound, as physically the aft ejector would restrict such 


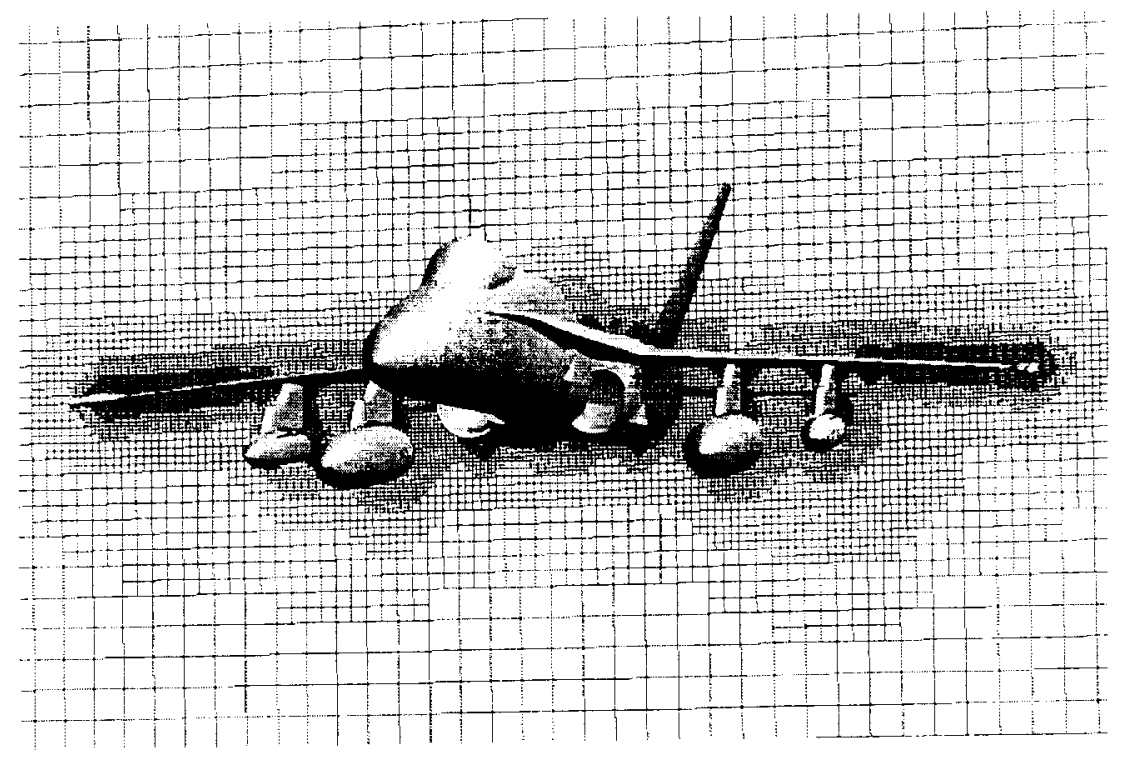

Figure 4: Cutting plane through the Cartesian volume mesh.

polyhedra.

In addition to mesh refinement near geometric features, pre-specified adaption regions are arranged around the major components of the F-18 aircraft to resolve the shock structures that occur at the current flow conditions. The adaption region which surrounds the JDAM translates with the center of mass (c.m.) location as the store drops. In the future, these pre-specified regions will be replaced with automated solution and geometry adaptation similar to the steady-state scheme outlined by Aftosmis and Berger[16]. A mesh refinement comparison was performed for the static, steady-state simulation with the JDAM in its initial position at the Mach 0.962 flight conditions (cf. Table 2). The resulting volume mesh is isotropic and contains $3.8 \mathrm{M}$ cells with a surface resolution of $1.0 \mathrm{in}$. A volume mesh cutting plane through the wing is shown in Fig. 4. Details of the mesh adaptation to the moving geometry will be presented in Sec. 3 .

\subsection{Flow Solver}

The inviscid, parallel multigrid flow solver of Aftosmis et al.[17] provides static, steady-state flow simulations for Cartesian meshes. Recently, this flow solver has been extended to provide capability for time-dependent flows, including dynamic simulations with rigid bodies in relative motion[18, 19]. The current work implements an independent 6-DOF module which can be utilized as a stand-alone external application, or tightly coupled within the time-dependent flow solver. A flow diagram for the 6-DOF/CFD simulation process is shown in Fig. 5. The XML4CFD interface[20] is utilized to integrate the independent mesh generation, flow solver, post-processing, and 6-DOF steps into a unified computational framework. 


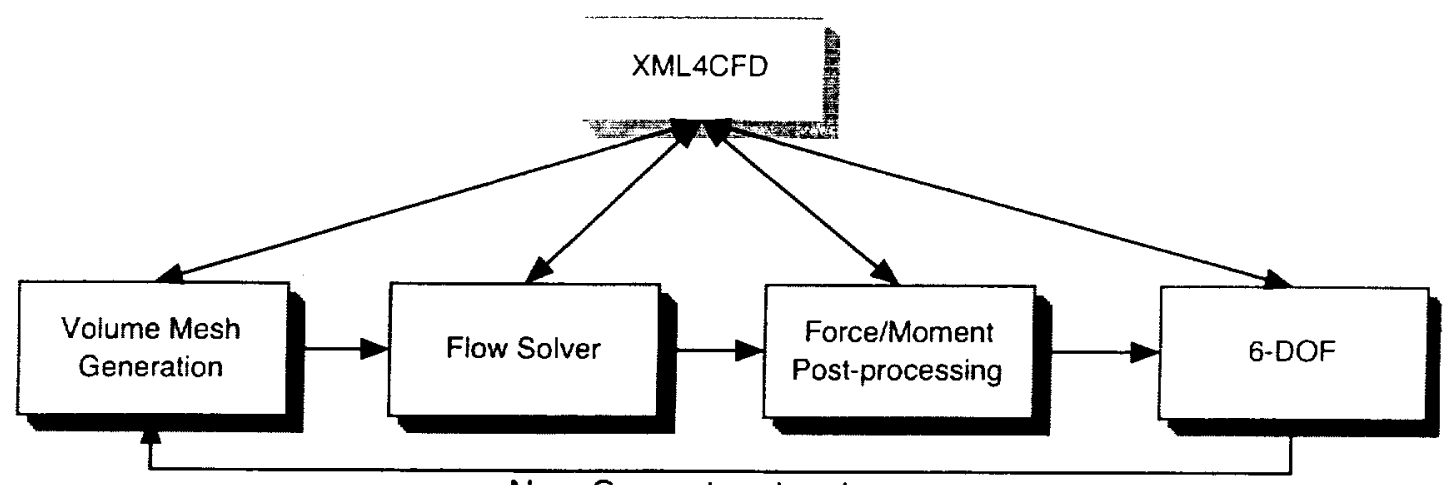

New Geometry at $n+1$

Figure 5: Process diagram for 6-DOF simulations. The red-colored processes are serial and the blue parallel. The XML4CFD interface[20] provides a single repository and API for the moving-body information required by the separate processes.

The 6-DOF module decomposes the rigid-body motion into a translation of the center of mass and a rotation about an axis passing through the c.m. location. The position of the c.m. is updated using Newton's laws of motion in the inertial frame, while the rotation of the body is determined by numerically integrating Euler's equations of motion in a body principal-axis system. The rotational position of the body is specified using Euler parameters, which are updated by numerical integration of the angular velocity. General external applied forces, in either the aerodynamic or body coordinate frames, can be specified. A detailed discussion of the 6-DOF model, along with validation test cases is presented in App. A.

\subsection{Ejector Force Model}

The JDAM is forced away from its wing pylon by means of identical piston ejectors located in the lateral plane of the store, -10.11 in. forward of the c.m., and 9.89 in. aft. The ejectors extend during operation for 6 in., and the force of each ejector is a polynomial function of this stroke extension (cf. Cenko[7]). As the store moves away from the pylon it begins to pitch and yaw due to aerodynamic forces, and the stroke length of the individual ejectors responds asymmetrically. This response of the ejectors to the store motion is modeled, and the result is presented as a function of time for each piston. This modeling process for the F-18/JDAM is described by Fortin et al.[14], and the results are presented in Table 1. A consistent theme with previous simulations is that this ejector model is limited (cf. $[8,9,14,15]$ ). Since even slight errors in the initial trajectory of the store can become augmented as the separation simulation is marched forward in time, researchers have modified either the ejector model, or the computed JDAM trajectory, in order to provide a realistic store separation. Physically, the JDAM is constrained by the ejector mechanism, which is not accounted for in simplistic models. For example, the JDAM cannot be allowed to pitch nose-down without bound, as physically the aft ejector would restrict such 
motions.

\begin{tabular}{||c||c|c||}
\hline Time(sec) & $\begin{array}{c}\text { Forward Ejector } \\
\text { Force (lbf) }\end{array}$ & $\begin{array}{c}\text { Aft Ejector } \\
\text { Force (lbf) }\end{array}$ \\
\hline \hline 0.00 & 97 & 97 \\
\hline 0.01 & 206 & 223 \\
\hline 0.02 & 531 & 283 \\
\hline 0.03 & 1053 & 549 \\
\hline 0.04 & 4723 & 988 \\
\hline 0.05 & 4641 & 4708 \\
\hline 0.06 & 4542 & 4633 \\
\hline 0.07 & 4414 & 4528 \\
\hline 0.08 & 4255 & 4386 \\
\hline 0.09 & 0 & 4243 \\
\hline 0.10 & 0 & 0 \\
\hline
\end{tabular}

Table 1: Modeled F/A-18C/GBU-32 ejector forces (cf. $[7,14]$ ).

While the focus of the current work is not to develop an ejector model for the F18/JDAM configuration, simulating the store separation with an ejector model which has known inaccuracies serves little purpose. An attempt to modify the ejector model to account for the constraint imposed by the wing pylon and ejector mechanism is proposed. First, following the a posteriori observations of Cenko[8], the magnitude of the ejector forces was increased by $25 \%$. Next, it is assumed that while the ejectors are accelerating (roughly $0.0<t<0.05 \mathrm{sec}$ ), the rotation of the JDAM is restricted by friction between the ejector pistons and the JDAM surface. From examining the flight data it is clear that the rotation is not completely restrained, so a friction resistance equivalent to $50 \%$ of the aerodynamic moments is imposed initially, which is allowed to linearly decrease to no resistance at $t=0.05 \mathrm{sec}$. This modified ejector model is used with all the simulation results presented here.

\section{Computed Results}

The numerical scheme outlined in the previous section was used to compute the separation of a GBU-32/JDAM from an F/A-18C at the two flight conditions listed in Table 2. The inertial properties for the JDAM were provided by the Navy, and are summarized in Table 3. The pylon ejector modeling was discussed in Sec. 2.3. This configuration was tested in the wind-tunnel using a Captive Trajectory System (CTS) and in-flight by the U.S. Navy (cf. Cenko[7]). Near sonic speeds, the variation of pitching and yawing moments experienced by the JDAM with Mach number becomes highly non-linear. This strong non-linearity makes trajectory prediction using linearized methods (cf. Keen[21]) challenging. High-fidelity CFD methods can potentially provide a cost-effective, accurate tool for predicting store trajectories at all 
flight conditions.

\begin{tabular}{||c||c|c||}
\hline & Case 1 & Case 2 \\
\hline \hline Mach number $\left(M_{\infty}\right)$ & 0.962 & 1.055 \\
\hline Altitude $(h)$ & $6332 \mathrm{ft}$. & $10,832 \mathrm{ft}$. \\
\hline AOA $(\alpha)$ & $0.46^{\circ}$ & $-0.65^{\circ}$ \\
\hline Dive Angle $(\gamma)$ & $43.0^{\circ}$ & $44.0^{\circ}$ \\
\hline
\end{tabular}

Table 2: Computed flight conditions.

\begin{tabular}{||c||c||}
\hline$S_{\text {ref }}$ & 1.767 sq. $\mathrm{ft}$. \\
\hline$L_{r e f}$ & $1.5 \mathrm{ft}$. \\
\hline c.m. & 62.66 in. from nose \\
\hline mass & $2059.44 \mathrm{lbm}$ \\
\hline$I_{x x}$ & 20.02 slug - sq. ft. \\
\hline$I_{y y}$ & 406.56 slug - sq. ft. \\
\hline$I_{z z}$ & 406.59 slug - sq. ft. \\
\hline$I_{x z}$ & -0.680 slug - sq. ft. \\
\hline$I_{x y}$ & 0.860 slug - sq. ft. \\
\hline$I_{y z}$ & 0.00 slug - sq. ft. \\
\hline
\end{tabular}

Table 3: GBU-32 JDAM inertial properties and reference quantities.

Static, steady-state simulations were computed with the JDAM in its initial position below the wing pylon for both flight conditions. Surface pressure contours on the body surface are shown in Fig. 6 for the $M_{\infty}=1.055$ simulation. The shock reflections on the wing pylons due to the stores are visible, as are the shocks that appear on the canopy, wing, and empennage. The cutting plane shows the resolution of the shocks to the farfield.

The computed forces and moments on the JDAM from the initial static simulations are compared with wind-tunnel and flight data in Table 4. No uncertainty predictions or error estimates are available for the wind tunnel or flight data. The computed results are in good agreement with the flight and tunnel data, with the largest discrepancy occurring in yawing moment at $M_{\infty}=0.962$, which is less than $10 \%$ variation. In general, the computed results compare more favorably to the fight data at $M_{\infty}=1.055$ than 0.962 , as would intuitively be expected.

\subsection{Sequential-static Simulations}

The current work simulates the separation of the JDAM using both time-dependent and steady-state methods. The inertia of the GBU-32 is very large, and the expectation is that unsteady effects are minimal, at least while the store is still close to the 

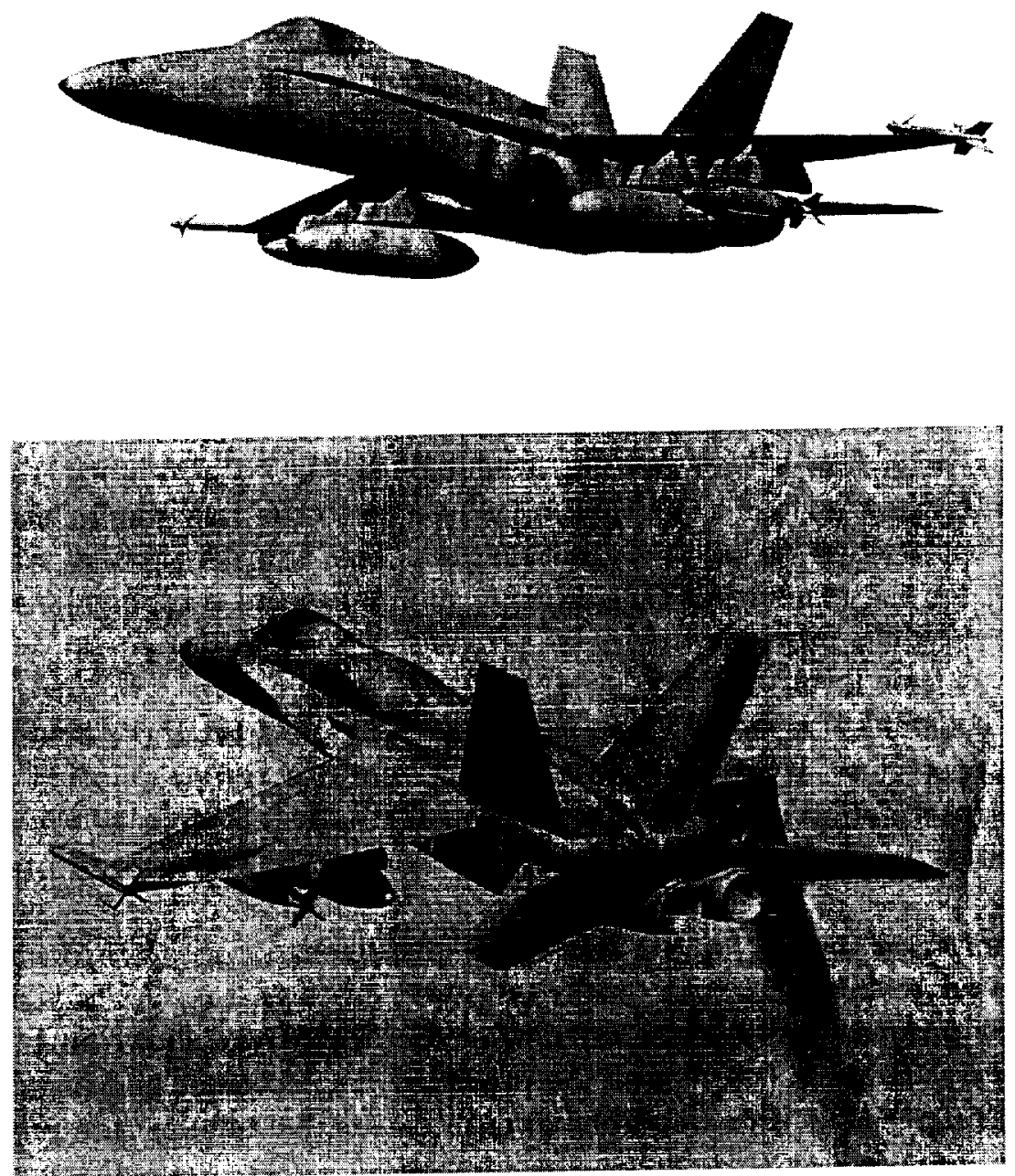

Figure 6: Surface pressure contours on the F-18C surface $\left(M_{\infty}=1.055, \alpha=-0.65^{\circ}\right)$.

pylon. This thesis is examined by comparison of time-dependent separation results with "sequential-static" simulations. The sequential-static results are presented first. In this method, the store is repositioned at the new time level based upon the computed loads at the previous time level (cf. Fig. 5, Sec. 2.2), however the flow solver ignores the motion of the body and treats it as a static, steady-state problem at the new body position. This approach can be attractive when accurate, time-dependent, 


\begin{tabular}{||c||c|c|c||c|c|c|}
\hline & $C_{A}$ & $C_{Y}$ & $C_{N}$ & $C_{l}$ & $C_{m}$ & $C_{n}$ \\
\hline \hline Wind Tunnel & - & 0.31 & 0.11 & - & -2.32 & -2.76 \\
\hline Flight & - & 0.31 & 0.15 & - & -2.5 & -2.8 \\
\hline Computed & 0.67 & 0.33 & 0.09 & 0.16 & -2.36 & -2.49 \\
\hline
\end{tabular}

a) $M_{\infty}=0.962, \alpha=0.46^{\circ}, \gamma=43^{\circ}$

\begin{tabular}{||c||c|c|c||c|c|c|}
\hline & $C_{A}$ & $C_{Y}$ & $C_{N}$ & $C_{l}$ & $C_{m}$ & $C_{n}$ \\
\hline Wind Tunnel & - & 0.24 & -0.02 & - & -2.07 & -2.56 \\
\hline Flight & - & 0.25 & -0.05 & - & -2.0 & -2.2 \\
\hline Computed & 0.65 & 0.28 & -0.03 & 0.15 & -2.02 & -2.11 \\
\hline
\end{tabular}

b) $M_{\infty}=1.055, \alpha=-0.65^{\circ}, \gamma=44^{\circ}$

Table 4: Computed forces and moments on the JDAM for the initial store position. Wind tunnel and flight data taken from Cenko[7].

moving-body flow solvers are not available. In the current work, the computed solution at the previous time level is transfered to the new mesh, after the body has been repositioned, to use as an initial guess. This transfer process, which is described in [19] for the time-dependent scheme, minimizes the computational cost since the solution at the previous time level provides a good initial guess for the solution at the next time level.

A constant timestep of $\Delta t=0.0075 \mathrm{sec}$. is used for these simulations. Due to time constraints, it was not possible to perform a time resolution study for these cases. Information travels roughly one JDAM body length in 12 timesteps using this resolution, which is felt to be reasonable. All simulations were run through $t=0.45$ sec.

Computed results for the relative displacement of the JDAM c.m. location are compared to flight data for both computed cases in Fig. 7. Similar plots for the angular position and angular velocity of the JDAM are shown in Figs. 8 and 9 respectively. Below $t=0.20 \mathrm{sec}$. the predicted displacement and angular position are in good agreement with the flight data, however the angular rate prediction has begun to degrade. At later times, the cumulative errors in angular position lead to a poorer agreement with the flight data, while the predicted displacement of the c.m. correlates well through the simulation. The accuracy of the current predictions is commensurate with previous computed results for this same configuration[9-15]. The degradation of the predicted angular orientation will be discussed in the next section with the time-dependent simulations results.

The miss distance, or the distance between the closest points on the JDAM and any other component of the aircraft, is presented in Fig. 10. While the predicted displacement and angular position are in good agreement with the flight data over the time interval presented, the miss distance underpredicts the separation between 


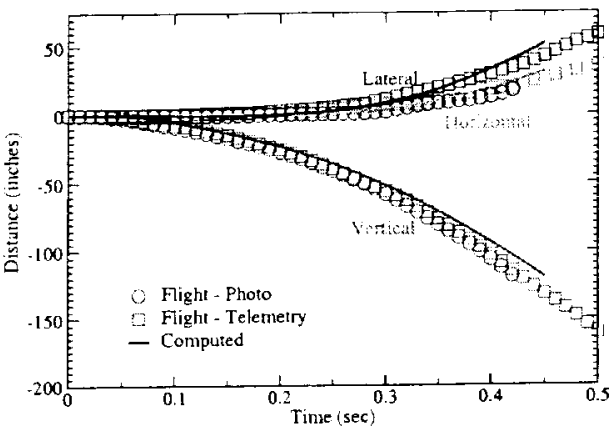

(a) $M_{\infty}=0.962, \alpha=0.46^{\circ}, \gamma=43^{\circ}$

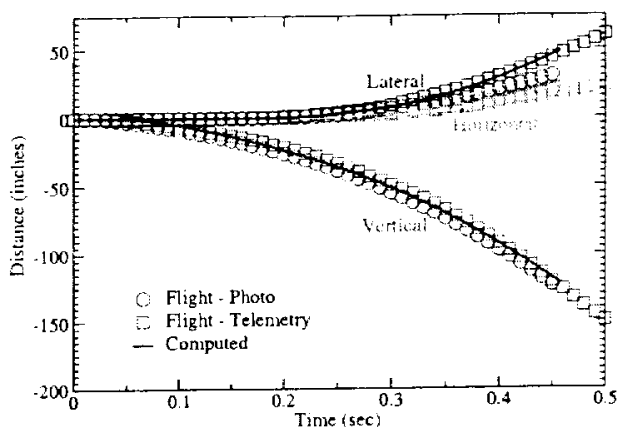

(b) $M_{\infty}=1.055, \alpha=-0.65^{\circ}, \gamma=44^{\circ}$

Figure 7: Relative displacement for sequential-static simulations. Flight data from Cenko[7].

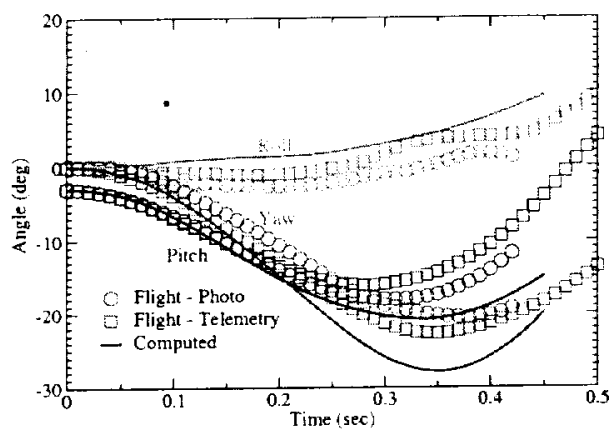

(a) $M_{\infty}=0.962, \alpha=0.46^{\circ}, \gamma=43^{\circ}$

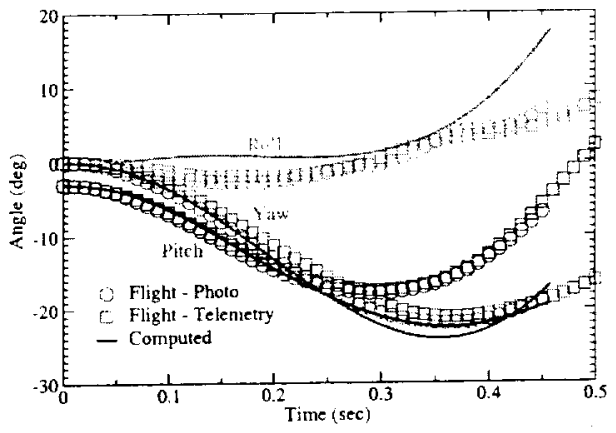

(b) $M_{\infty}=1.055, \alpha=-0.65^{\circ}, \gamma=44^{\circ}$

Figure 8: Angular positions for sequential-static simulations. Flight data from Cenko[7].

the store and the wing pylon. The explanation for this is that as the ejectors push away the store, there is a reaction force applied to the pylon. This reaction leads to a rolling moment on the aircraft which rolls the pylon away from the JDAM, i.e. increases the miss distance between the two. This reaction of the aircraft is not modeled in the current work (or in previous work in the literature), and hence the separation is underpredicted. The closest miss, which occurs near $t=0.10 \mathrm{sec}$., is caused by the tail fins sweeping under the pylon as the JDAM yaws nose outboard. At $t=0.20 \mathrm{sec}$. the closest component changes from the pylon to the EFT, as the body continues to yaw and fall.

Figure 11 shows a series of snapshots of the surface pressure as the JDAM falls 


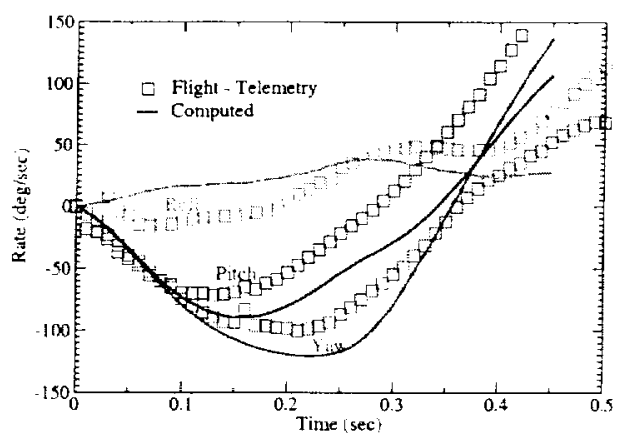

(a) $M_{\infty}=0.962, \alpha=0.46^{\circ}, \gamma=43^{\circ}$

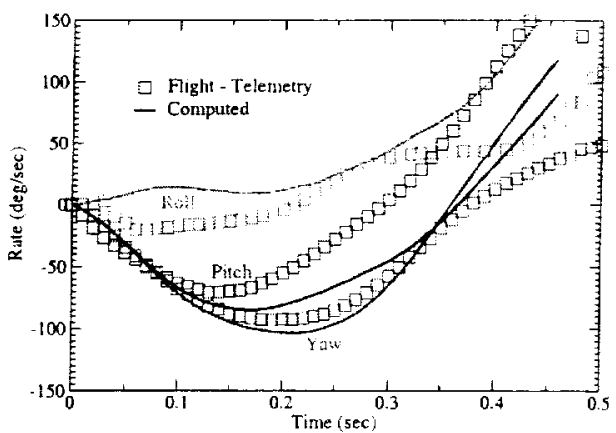

(b) $M_{\infty}=1.055, \alpha=-0.65^{\circ}, \gamma=44^{\circ}$

Figure 9: Angular rates for sequential-static simulations. Flight data from Cenko[7].

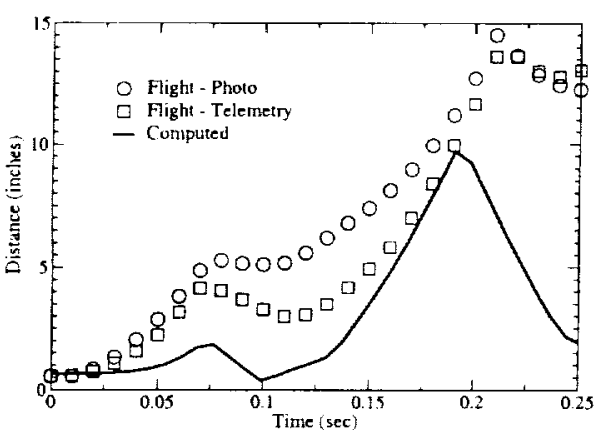

(a) $M_{\infty}=0.962, \alpha=0.46^{\circ}, \gamma=43^{\circ}$

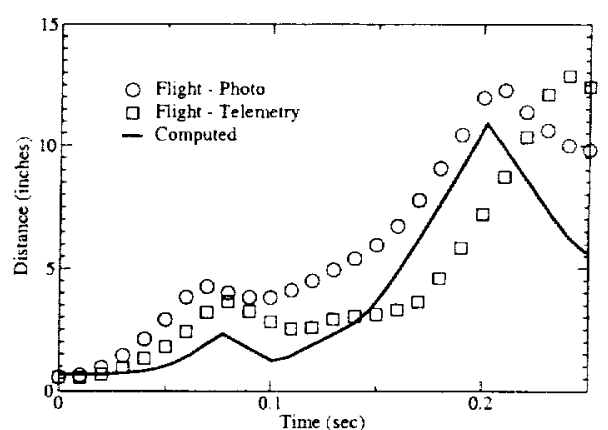

(b) $M_{\infty}=1.055, \alpha=-0.65^{\circ}, \gamma=44^{\circ}$

Figure 10: Miss distances for sequential-static simulations. Flight data from Cenko[7].

through $t=0.40 \mathrm{sec}$. in the $M_{\infty}=1.055$ simulation. The nose of the store is forced downward and outboard by the shock from the leading-edge of the wing. This causes the JDAM to pitch and yaw immediately upon release from the holding pylon. The change in shock structure on the pylon as the JDAM releases can be seen, as well as changes on the aft portion of the aircraft fuselage. As the JDAM falls, the tail fins provide restoring moments which cause the store pitch back nose up and inboard (compare with Figs. 8 and 9). A complementary series of snapshots which show the adaptation of the mesh to the moving geometry are shown in Fig. 12. The mesh automatically adapts to the new geometry position, and also coarsens in regions the body has moved through. 

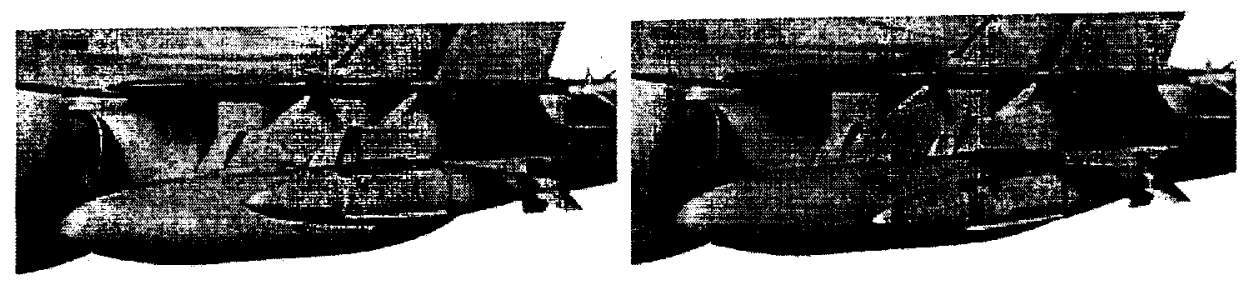

(a) $t=0.0 \mathrm{sec}$.

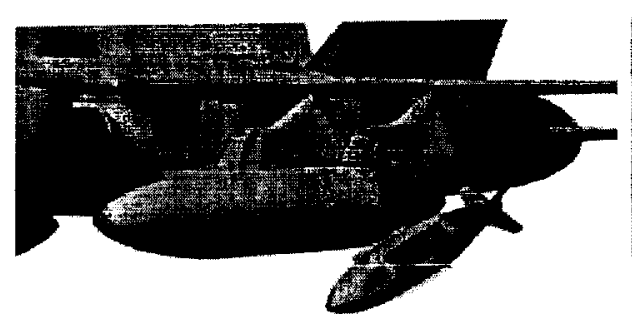

(c) $t=0.2 \mathrm{sec}$ (b) $t=0.1 \mathrm{sec}$.

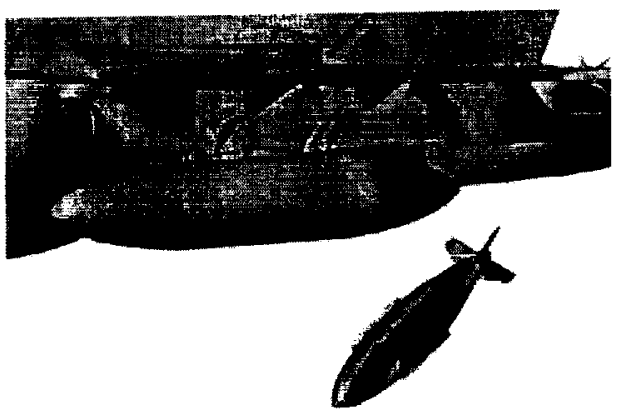

(d) $t=0.3 \mathrm{sec}$.

Figure 11: Surface pressure contours during JDAM separation.

\subsection{Time-dependent Simulations}

The previous section presented results of coupled 6-DOF/CFD trajectory predictions using sequential-static flow simulations. This is contrasted here with fullycoupled, time-dependent trajectory simulations performed using the Cartesian movingbody solver described in [19]. Analyzing Fig. 9, the angular rate prediction for the sequential-static simulations begins to degrade after the rotation of the body experiences both the highest velocities and an inflection point in the acceleration, i.e. near $t=0.125$ for pitch rate, and $t=0.20$ for yaw rate. This combination of high velocity and change in sign of acceleration indicate regions in the store trajectory where dynamic, or unsteady effects, may be significant. This is examined in Figs. 13-16, which present relative displacement, angular orientation, angular rate, and miss distance for the time-dependent simulations, compared with the sequential-static simulations and flight-test data. The data shows that the two CFD trajectory simulations are in good agreement prior to $t=0.125$, when the pitch rate reaches a maximum. After this point, the predicted pitch behavior is improved, however the yaw prediction degrades. The pitch and yaw trajectories are similar in the sequential-static and time-dependent 


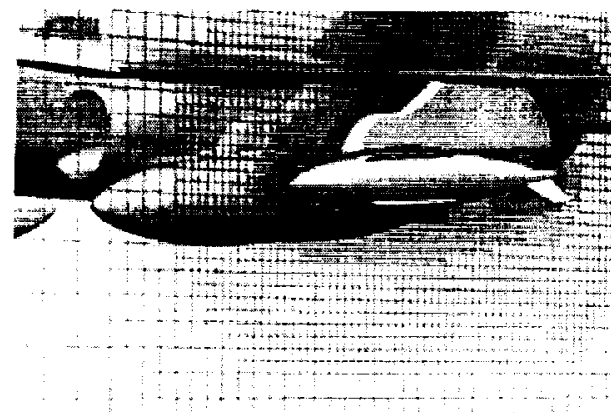

(a) $t=0.0 \mathrm{sec}$.

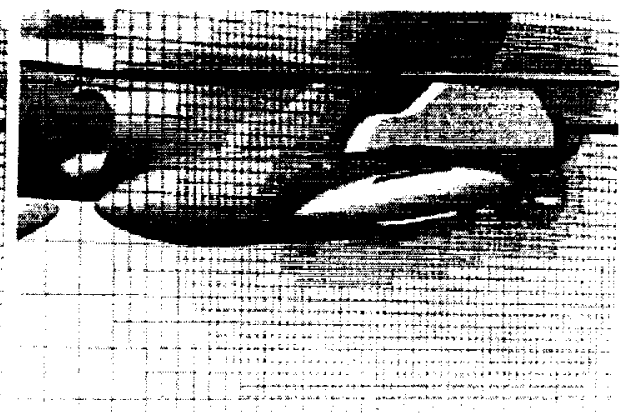

(b) $t=0.1 \mathrm{sec}$.

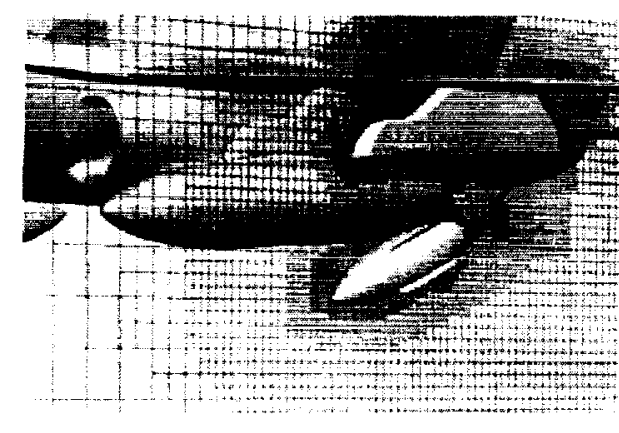

(c) $t=0.2 \mathrm{sec}$.

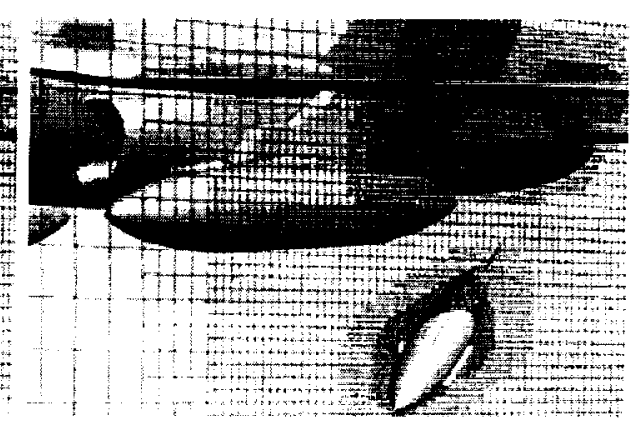

(d) $t=0.3 \mathrm{sec}$.

Figure 12: Cutting planes through the volume mesh during JDAM separation.

simulations, except for the response near the maximum rates, i.e. the dynamic effects are largely localized to this region of the trajectory. The relative displacement prediction is nearly unchanged in the time-dependent simulations at $M_{\infty}=1.055$, however $M_{\infty}=0.962$ shows a relatively significant change in vertical drop, which is not currently well understood. The underprediction of the separation distance after $t=0.20$ is caused by the over-predicted yaw angle in both the sequential-static and time-dependent simulations, which causes the tail fins to remain close to the EFT.

Consistently, in both the sequential-static and time-dependent simulations, the predicted roll behavior of the JDAM does not correlate well with the flight data. This is not unique to the current work, and has been noted in previous trajectory predictions for this configuration[7-15]. Cenko[7] notes "[roll attitude] is the hardest to predict, fortunately has a minimal impact on the trajectory". While it's true that small changes in roll orientation are likely insignificant, the current predictions consistently vary from the flight data by roughly $5^{\circ}$ of roll, and even while the store is still being pushed by the ejectors the roll is predicted in the opposite direction. Since the roll orientation can effect the restoring moment provided by the tail fins, it's unclear whether these small differences can accumulate to produce the larger errors 


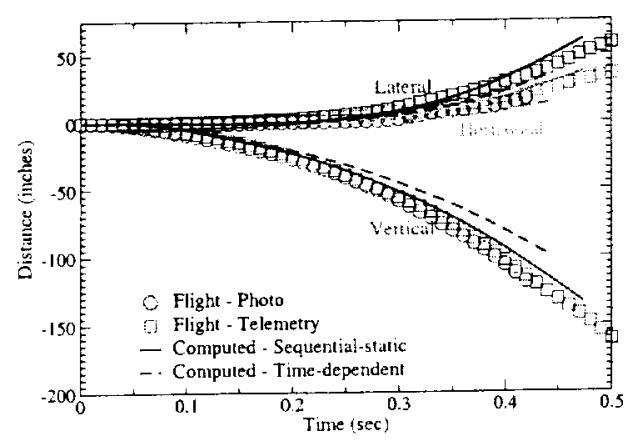

(a) $M_{\infty}=0.962, \alpha=0.46^{\circ}, \gamma=43^{\circ}$

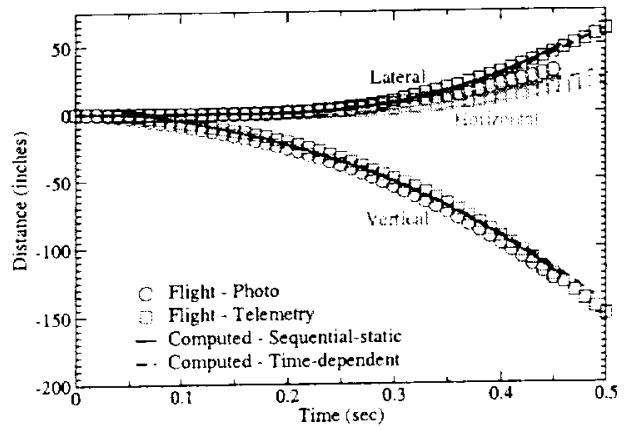

(b) $M_{\infty}=1.055, \alpha=-0.65^{\circ}, \gamma=44^{\circ}$

Figure 13: Relative displacement for time-dependent simulations. Flight data from Cenko[7].

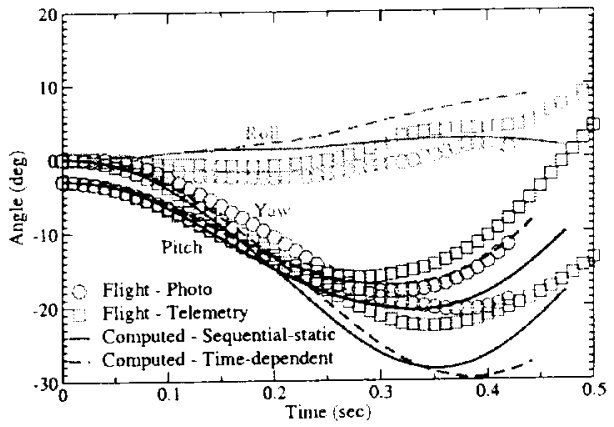

(a) $M_{\infty}=0.962, \alpha=0.46^{\circ}, \gamma=43^{\circ}$

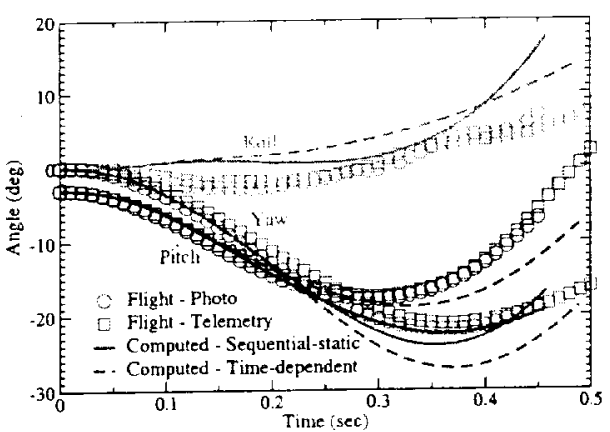

(b) $M_{\infty}=1.055, \alpha=-0.65^{\circ}, \gamma=44^{\circ}$

Figure 14: Angular positions for time-dependent simulations. Flight data from Cenko[7].

seen in pitch and yaw prediction in the current work.

\subsection{Computational Cost}

The computational cost for the current Cartesian/6-DOF scheme is presented in two forms; absolute and relative to computing a fixed "database" of static results. Note that the current work was performed with tools designed for computing a single fixed static simulation, and little effort has gone into tailoring them for sequential

moving-body calculations. All simulated results presented here were computed using NASA Ames' $1024 \mathrm{CPU}$, single-image SGI Origin 3000 (O3K) which has $600 \mathrm{MHz}$ 


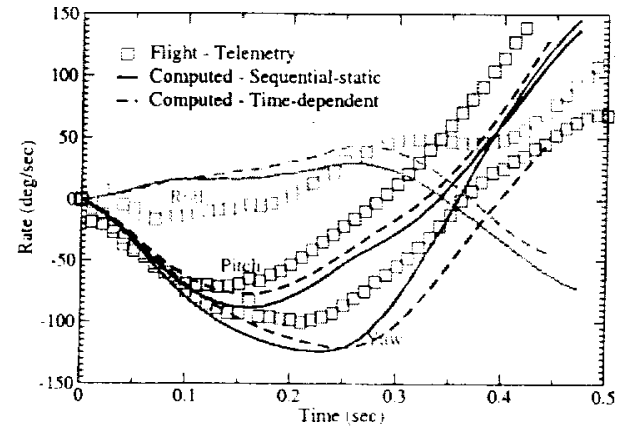

(a) $M_{\infty}=0.962, \alpha=0.46^{\circ}, \gamma=43^{\circ}$

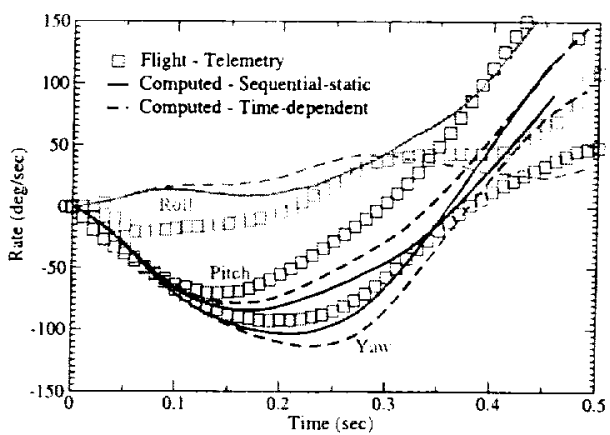

(b) $M_{\infty}=1.055, \alpha=-0.65^{\circ}, \gamma=44^{\circ}$

Figure 15: Angular rates for time-dependent simulations. Flight data from Cenko[7].

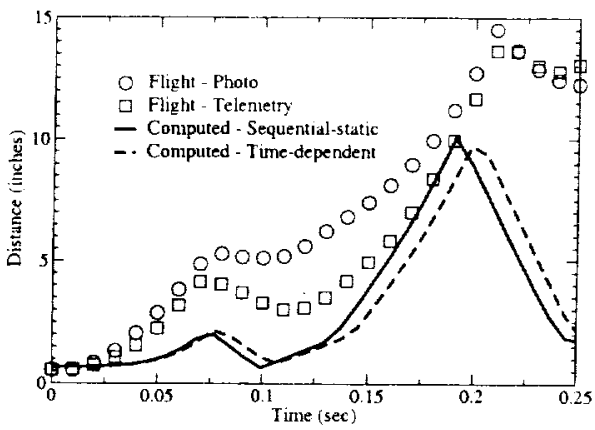

(a) $M_{\infty}=0.962, \alpha=0.46^{\circ}, \gamma=43^{\circ}$

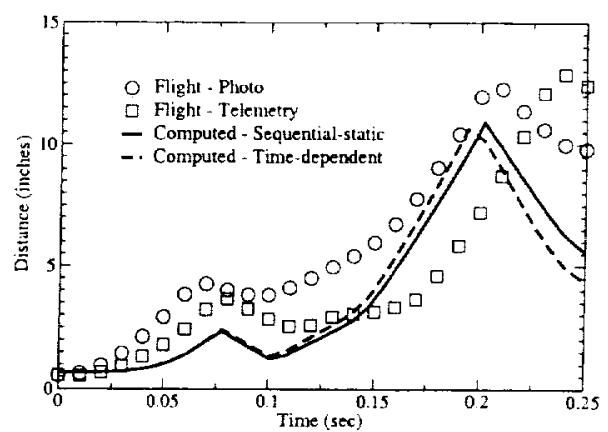

(b) $M_{\infty}=1.055, \alpha=-0.65^{\circ}, \gamma=44^{\circ}$

Figure 16: Miss distances for time-dependent simulations. Flight data from Cenko[7].

MIPS4 processors. The current flow solver has been demonstrated to scale linearly to 512 CPUs on this architecture for problems of the size considered here. The current simulations all required roughly 260 single-CPU-hours of computational time to complete, with less than $5 \%$ of the computational time utilized by the volume mesh generation process. The sequential-static and time-dependent simulations require the same computational time with the current scheme. The wallclock time to complete a simulation using 32 CPUs is approximately 15 hours. This time reflects the adverse effects of the serial mesh generation on the parallel efficiency of the entire process. Parallelizing the entire process, including volume mesh generation, will be a major focus of future work. 
The current work couples together the CFD flow solver and the 6-DOF trajectory prediction. Another method of integrating high-fidelity CFD with 6-DOF predictions is to build a computational database of results, and then "fly" 6-DOF trajectories through this computed database. The advantage of this approach is that once the initial database is created, many 6-DOF trajectories can be computed essentially at no cost. The disadvantage of the database approach for 6-DOF simulations is the large number of computational cases required to build even a minimal database. For a single fixed wind vector $\left(M_{\infty}, \alpha, \beta\right)$ there are 6 free parameters ( 3 displacement and 3 angular position) for a static CFD database. ${ }^{*}$ If each of these is allowed to vary over 10 distinct states (which is relatively coarse), then $10^{6}$ computed cases are required to fill the database. This is impractical even for wind tunnel programs. It's possible to reduce the required independent variables by assuming that the horizontal and lateral relative displacements are much less than the vertical, and that the roll orientation of the body can be ignored. This reduction leaves on the order of 1000 data points required for steady-state simulation. In the current work, an initial steady-state calculation is required at the initial position of the store, and each timestep costs roughly $1 / 5$ of a full static simulation. As 60 steps were required for a full simulation using the current timestep, the cost for the current coupled 6-DOF trajectory simulations is roughly 10 steady, static simulations. This implies that on the order of 100 such coupled simulations can be performed for the cost of building a coarse, approximate database. Further, each coupled simulation is independent, so that the simulations can be carried out in parallel. The higher accuracy and relatively low cost makes these coupled CFD/6-DOF simulations an attractive analysis tool.

\section{Summary}

The utility of a coupled Cartesian/6-DOF trajectory prediction scheme has been demonstrated by simulation of a GBU-32 JDAM separating from an F/A-18C. The Cartesian scheme provides an automated, robust meshing scheme which can easily be integrated into a design analysis. The accuracy and computational cost of the current simulated results are commensurate with previous results for the F-18/JDAM separation computed using body-fitted approaches.

Future work will progress on two major fronts; understanding the discrepancies in predicted angular orientation that occur at later time levels, and optimizing the flow simulation process for these moving-body simulations. There are many possible explanations, both computational and experimental, for the degradation in the predicted trajectory at later time levels. It's important to understand whether this behavior is related to the current approach so that it can be corrected, if necessary. The process optimization itself will mainly focus on parallelizing the volume mesh generation, and incorporating an solution-adaptive capability.

* Static here refers to the absence of any dynamic stability derivative information. 


\section{Acknowledgments}

The authors would like to thank Dr. Alex Cenko of the U.S. Navy for his help in acquiring the geometry, and Dr. Ralph Noack of the Univ. of Alabama, Birmingham for his assistance throughout this project. John Guse of Boeing generously supplied information regarding the inertial properties of the F-18C. Marsha Berger was supported in part by AFOSR grant F19620-00-0099.

\section{References}

[1] Meakin, R. L., "Computations of the Unsteady Flow About a Generic Wing/Pylon/Finned-Store Configuration," AIAA Paper 92-4568-CP, Aug. 1992.

[2] Prewitt, N.C., Belk, D., and Shyy, W., "Multiple Body Trajectory Calculations Using the Beggar Code," AIAA Paper 99-0913, Jan. 1999.

[3] Löhner, R. and Baum, J.D., "Three-Dimensional Store Separation Using a Finite Element Solver and Adaptive Remeshing," AIAA Paper 91-0602, Jan. 1991.

[4] Cavallo, P.A. and Dash, S.M., "Aerodynamics of Multi-Body Separation Using Adaptive Unstructured Grids," AIAA Paper 2000-4407, Aug. 2000.

[5] Welterlen, T.J. and Leone, C., "Application of Viscous Cartesian CFD to Aircraft Store Carriage and Separation Simulation," AIAA Paper 96-2453, June 1996.

[6] Aftosmis, M.J., Berger, M.J., and Melton, J.E., "Robust and Efficient Cartesian Mesh Generation for Component-Based Geometry," AIAA Paper 97-0196, Jan. 1997. Also AIAA Joumal 36(6):952-960,June 1998.

[7] Cenko, A., "F-18/JDAM CFD Challenge Wind Tunnel Flight Test Results," AIAA Paper 99-0120, Jan. 1999.

[8] Cenko, A., Lutton, M., and Tutty, M., "F/A-18/C/JDAM Applied Computational Fluid Dynamics Challenge II Results," AIAA Paper 2000-0795, Jan. 2000.

[9] Noack, R.W. and Jolly, B., "Fully Time Accurate CFD Simulations of JDAM Separation from an F-18C Aircraft," AIAA Paper 2000-0794, Jan. 2000.

[10] Welterlen, T.J., "Store Release Simulation on the F/A-18C Using Splitflow," AIAA Paper 99-0124, Jan. 1999.

[11] Tomaro, R.F., Witzeman, F.C., and Strang, W.Z., "A Solution on the F-18C for Store Separation Simulation Using Cobalt60," AIAA Paper 99-0122, Jan. 1999.

[12] Woodson, S.H. and Brunner, C.W.S., "Analysis of Unstructured CFD Codes for Accurate Prediction of Aircraft Store Trajectories," AIAA Paper 99-0123, Jan. 1999. 
[13] Fairlie, B.D. and Caldeira, R.H., "Prediction of JDAM Separation Characteristics from the F/A-18C Aircraft," AIAA Paper 99-0126, Jan. 1999.

[14] Fortin, F., Benmeddour, A., Jones, D.J, "Application of the Canadian Code to the F/A-18C JDAM Separation," AIAA Paper 99-0127, Jan. 1999.

[15] Sickles, W.L., Denny, A.G., and Nichols, R.H., "Time-Accurate Predictions of the JDAM Separation from an F-18C Aircraft," AIAA Paper 2000-0796, Jan. 2000 .

[16] Aftosmis, M.J. and Berger, M.J., "Multilevel Error Estimation and Adaptive h-Refinement for Cartesian Meshes with Embedded Boundaries," AIAA Paper 2002-0863, June 2002.

[17] Aftosmis, M.J., Berger, M.J., and Adomavicius, G., "A Parallel Multilevel Method for Adaptively Refined Cartesian Grids with Embedded Boundaries," AIAA Paper 2000-0808, Jan. 2000.

[18] Murman, S.M., Aftosmis, M.J., and Berger, M.J., "Numerical Simulation of Rolling-Airframes Using a Multi-Level Cartesian Method," AIAA Paper 20022798, June 2002.

[19] Murman, S.M., Aftosmis, M.J., and Berger, M.J., "Implicit Approaches for Moving Boundaries in a 3-D Cartesian Method," AIAA Paper 2003-1119, Jan. 2003.

[20] Murman, S.M., Chan, W.M., Aftosmis, M.J., and Meakin, R.L., "An Interface for Specifying Rigid-Body Motion for CFD Applications," AIAA Paper 20031237, Jan. 2003.

[21] Keen, K.S., "New Approaches to Computational Aircraft/Store Weapons Integration," AIAA Paper 90-0274, Jan. 1990. 


\section{Appendix}

\section{A 6-DOF Model}

This appendix describes an implementation of the unconstrained motion of a rigid body, commonly referred to as six-degree-of-freedom (6-DOF) motion. The 6DOF model is implemented as a stand-alone package with a well-defined Application Programming Interface (API). In this manner it can easily be integrated within a CFD flow solver, or similar application, or used as a stand-alone package, for example when performing trajectory simulations within a pre-existing database of force and moment data.

The 6-DOF motion is computed by solving the Newton-Euler equations for rigidbody motion. The motion is broken into a translation of the center of mass (c.m.) of the body (Newton's equations), and a rotation about a centroidal axis system attached to the body (Euler's equations) (cf. Fig.A.1). Here superscripts are used to designate the coordinate system, with $i$ referring to the inertial frame, and $b$ the body frame. The inertial frame is considered to be the natural coordinate system of the geometry. Note that this inertial frame is not in general identical to the aerodynamic frame in which forces and moments are calculated, so a transformation from the aerodynamic frame to the inertial frame is required.

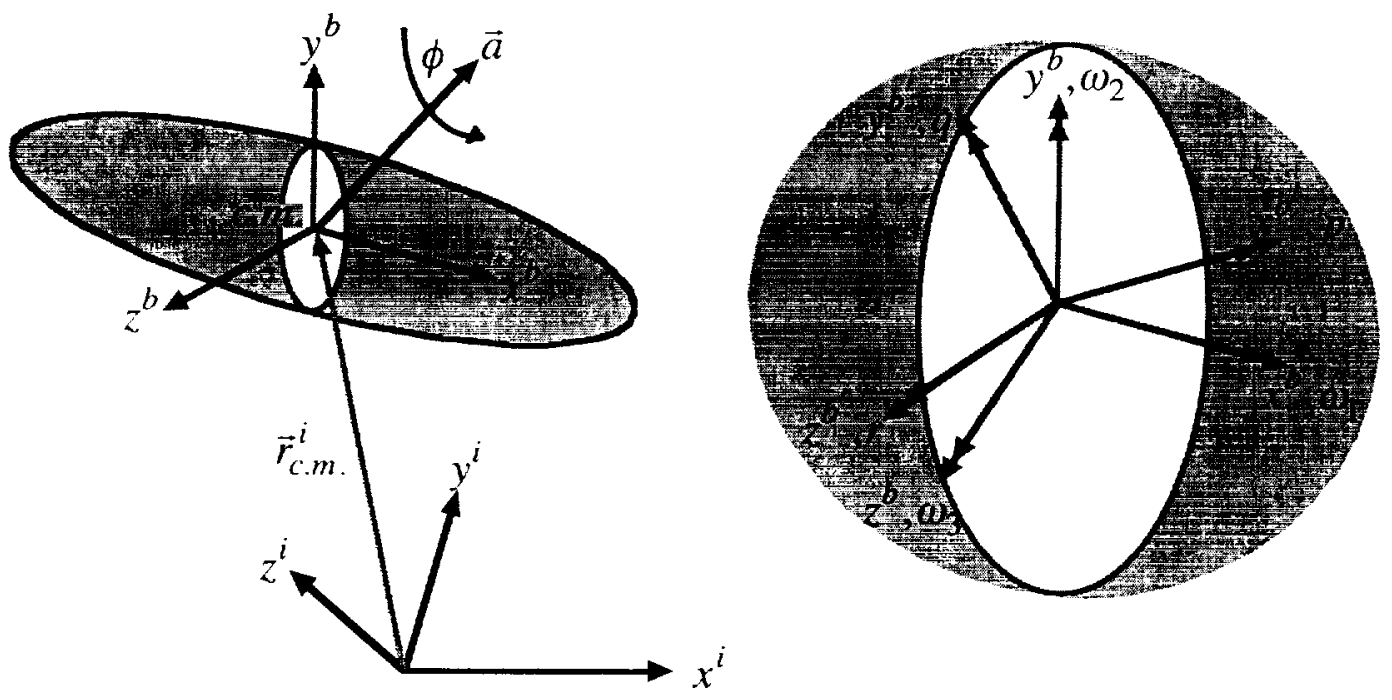

Figure A.1: Inertial and body-fixed coordinate systems. Superscripts are used to designate the coordinate system, with $i$ referring to the inertial frame, and $b$ the body frame. The body frame is rotated by an angle $\phi$ about the axis a relative to the inertial frame. The body frame is the unique frame defined by the principal axes of the moments of inertia. This is contrasted with the non-unique body frame $b^{\prime}$ which is defined by convenience. The angular velocity is $\boldsymbol{\omega}$ is the principal axes frame, and $(p, q, r)$ in the general body frame.

The mass center translation is governed by Newton's laws of motion, which are 
written in the inertial frame as

$$
\mathbf{F}^{i}=\mathbf{F}^{i}+\mathbf{F}_{e}^{i}+\mathbf{F}_{g}^{i}=m \ddot{\mathbf{r}}_{c \cdot m}^{i}
$$

where the applied force acting through the center of mass has been broken into three components; the aerodynamic forces $\mathbf{F}_{a}^{i}$, the external applied forces (such as thrust) $\mathbf{F}_{e}^{i}$, and the forces due to gravity $\mathbf{F}_{g}^{i}$. Equation $A .1$ is written in non-dimensional variables using the reference density $\left(\rho_{\infty}\right)$, reference velocity (freestream sonic speed $\left.a_{\infty}\right)$, and a reference length $(L)$. The non-dimensional mass is thus the dimensional mass scaled by the mass contained in a reference unit volume

$$
m=\frac{\tilde{m}}{\rho_{\infty} L^{3}}
$$

and similary the forces and gravity are non-dimensionalized by

$$
\begin{gathered}
\mathbf{F}=\frac{\tilde{\mathbf{F}}}{\rho_{\infty} a_{\infty}^{2} L^{2}} \\
\mathbf{g}=\frac{\tilde{\mathbf{g}} L}{a_{\infty}^{2}}
\end{gathered}
$$

Newton's laws can be integrated directly to give the position of the mass center as a function of time. Holding $\mathbf{F}$ constant over the discrete physical timestep $\left(t^{n}, t^{n+1}\right)$ gives

$$
\mathbf{r}_{c . m .}^{i}\left(t^{n+1}\right)=\frac{1}{2} \frac{\mathbf{F}^{i}}{m} \Delta t^{2}+\mathbf{u}_{c . m .}^{i}\left(t^{n}\right) \Delta t+\mathbf{r}_{c . m .}^{i}\left(t^{n}\right)
$$

where $\mathbf{u}_{c . m \text {. }}^{i}$ is the velocity of the center of mass.

The rotational motion is governed by Euler's equations of motion. The body axes are specified to coincide with the principal axes of inertia, with origin at the center of mass (cf. Fig. A.1). Euler's equations are then

$$
\begin{aligned}
& M_{1}^{b}=I_{1}^{b} \dot{\omega}_{1}^{b}-\left(I_{2}^{b}-I_{3}^{b}\right) \omega_{2}^{b} \omega_{3}^{b} \\
& M_{2}^{b}=I_{2}^{b} \dot{\omega}_{2}^{b}-\left(I_{3}^{b}-I_{1}^{b}\right) \omega_{3}^{b} \omega_{1}^{b} \\
& M_{3}^{b}=I_{3}^{b} \dot{\omega}_{3}^{b}-\left(I_{1}^{b}-I_{2}^{b}\right) \omega_{1}^{b} \omega_{2}^{b}
\end{aligned}
$$

where $\mathbf{M}^{b}$ are the applied moments in the body frame, and are broken into aerodynamic and external components as in Eqn.A.1. $\omega^{b}$ is the angular velocity in the body frame, and $\mathbf{I}^{b}$ are the principal moments of inertia. Using the same reference quantities as above, the non-dimensional applied moments and moments of inertia are given by

$$
\begin{aligned}
\mathbf{M}^{b} & =\frac{\tilde{\mathbf{M}}^{b}}{\rho_{\infty} a_{\infty}^{2} L^{3}} \\
\mathbf{I}^{b} & =\frac{\tilde{\mathbf{I}}^{b}}{\rho_{\infty} L^{5}}
\end{aligned}
$$


Equation A.3 is integrated numerically using a $4^{\text {th }}$-order Runge-Kutta scheme.

In order to transform the angular velocity into a change in orientation, it's desirable to use quaternions, commonly referred to as "Euler parameters", to specify the angular orientation of the body frame with respect to the inertial frame (cf. Fig. A.1). A quadratic transformation matrix $\mathbf{A}$, which is composed of the direction cosines, can be expressed as the result of two successive linear transformations

$$
\mathbf{A}=\mathbf{G} \mathbf{L}^{T}
$$

where $\mathbf{G}$ and $\mathbf{L}^{T}$ are both composed of the 4 Euler parameters

$$
\mathbf{p}=\left[\begin{array}{llll}
e_{0} & e_{1} & e_{2} & e_{3}
\end{array}\right]^{T}
$$

The transformation matrix in terms of the Euler parameters is given by

$$
\mathbf{A}=2\left[\begin{array}{lll}
e_{0}^{2}+e_{1}^{2}-\frac{1}{2} & e_{1} e_{2}-e_{0} e_{3} & e_{1} e_{3}+e_{0} e_{2} \\
e_{1} e_{2}+e_{0} e_{3} & e_{0}^{2}+e_{2}^{2}-\frac{1}{2} & e_{2} e_{3}-e_{0} e_{1} \\
e_{1} e_{3}-e_{0} e_{2} & e_{2} e_{3}+e_{0} e_{1} & e_{0}^{2}+e_{3}^{2}-\frac{1}{2}
\end{array}\right]
$$

The Euler parameters specify an axis of rotation (a), and an angular displacement about that axis $(\phi)$

$$
\begin{aligned}
& e_{0}=\cos \frac{\phi}{2} \\
& \epsilon_{1}=a_{x} \sin \frac{\phi}{2} \\
& e_{2}=a_{y} \sin \frac{\phi}{2} \\
& e_{3}=a_{z} \sin \frac{\phi}{2}
\end{aligned}
$$

According to Euler's theory of motion, the Euler parameters are the same in both the body and fixed reference frames, so no superscript appears on $\mathbf{p}$, however note that in this case the discussion assumes the reference frame is attached to the center of mass.

Using this, the change in orientation due to rotation can be found through

$$
\dot{\mathbf{p}}=\frac{1}{2} \mathbf{L}^{T} \boldsymbol{\omega}^{b}
$$

which can also be integrated numerically using a $4^{\text {th }}$-order Runge-Kutta scheme. Since the Euler parameters are unit-normalized quaternions, it's necessary to impose that $|\mathbf{p}|=1$ after solving Eqn. A.6. $\mathbf{L}^{T}$ is given by

$$
\mathbf{L}^{T}=\left[\begin{array}{ccc}
-e_{1} & -e_{2} & -e_{3} \\
e_{0} & -e_{3} & e_{2} \\
e_{3} & e_{0} & -e_{1} \\
-e_{2} & e_{1} & e_{0}
\end{array}\right]
$$


In order to update the position of an unconstrained rigid body, the following procedure is thus followed

\section{Translate}

Solve Newton's law's of motion using Eqn. A.2 for the translation of the center of mass.

\section{Rotate}

\section{(a) Angular Velocity} Numerically solve Euler's equations of motion using Eqn. A.3 for the angular velocity in the body frame.

\section{(b) Euler Parameters}

Update the orientation of the body by numerically integrating Eqn. A.6 for the Euler parameters.

\section{Reposition}

Position the body according $\mathbf{r}^{i}=\mathbf{r}_{c . m}^{i}+\mathbf{A r}^{b}$

While quaternions are convenient for calculating the angular position of a rigid body, they are not always intuitive. It's often desirable to transform the quaternions to a set of three angles which are non-unique, but in practice often unambiguous. The Euler parameters can be converted to angular displacement $\left(\phi_{x}, \phi_{y}, \phi_{z}\right)$ of the body relative to the inertial frame using

$$
\begin{aligned}
\tan \left(\phi_{x}\right) & =\frac{2.0\left(e_{0} e_{1}+e_{2} e_{3}\right)}{e_{0}^{2}-e_{1}^{2}-e_{2}^{2}+e_{3}^{2}} \\
\sin \left(\phi_{y}\right) & =-2.0\left(e_{1} e_{3}-e_{0} e_{2}\right) \\
\tan \left(\phi_{z}\right) & =\frac{2.0\left(e_{1} e_{2}+e_{0} e_{3}\right)}{e_{0}^{2}+e_{1}^{2}-e_{2}^{2}-e_{3}^{2}}
\end{aligned}
$$

The potential singularity in $\mathrm{x}$ and $\mathrm{z}$ orientation is obvious.

\section{A.1 6-DOF Model Verification}

The 6-DOF implementation was verified using a variety of analytic test cases. Integration of Newton's laws (Eqn. A.1) is verified using response of a point mass to a constant external force, and the terminal velocity of a falling sphere examines this integration for a non-constant external force. Integration of Euler's Eqn. A.3 was examined using the response of a cylinder undergoing a coupled spin. A tumbling rectangular volume demonstrates that the numerical implementation has the same stability properties as the physical system.

Translation is integrated analytically according to Eqn. A.1, holding the applied force constant over the timestep. Since the integration is analytic, it is exact in the 
presence of a constant external force. To verify this consider a point mass with an initial upward velocity in a gravitational field. The force due to gravity is normalized such that $m \mathrm{~g}=(0,0,-1)$, and the initial velocity vector is $\mathbf{u}=(0,0,1)$. Figure A.2 shows the exact solution for this case compared with the computed solutions taken with $\Delta t=0.025$ and $\Delta t=0.1$. Since the integration is exact for this constant external force, the numerical integration reproduces the exact solution at both timesteps.

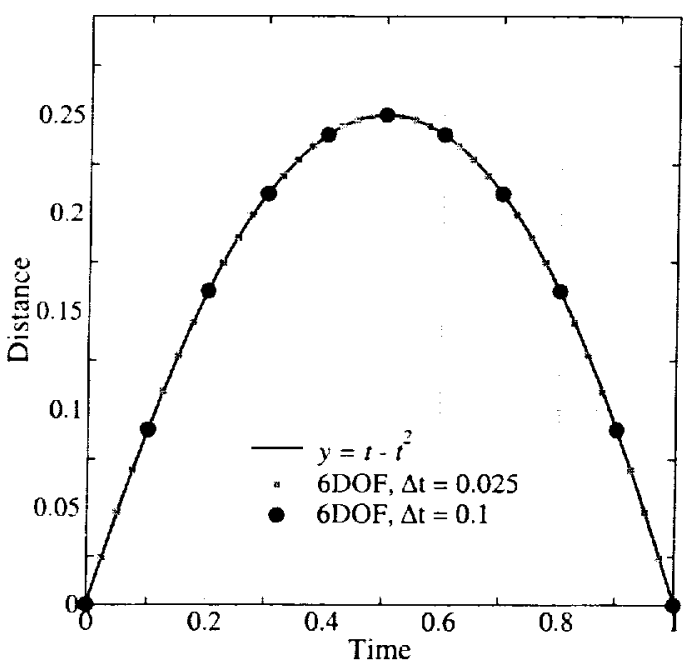

Figure A.2: Distance as a function of time for a unit point mass in a gravitational field. The force due to gravity is normalized such that $m \mathbf{g}=(0,0,-1)$, and the initial velocity vector is $\mathbf{u}=(0,0,1)$.

When the external force is non-constant, holding $\mathbf{F}$ fixed over the timestep results in formal first-order accuracy. To demonstrate this, consider a sphere with drag coefficient $C_{D}=0.5$ falling through air in a gravitational field. The external force is $\mathbf{F}=m \mathbf{g}-C_{D} \frac{1}{2} \rho u_{z}^{2} S$. When the gravity and drag forces balance each other the sphere reaches its terminal velocity $u_{\infty}=\sqrt{\frac{2 m g_{z}}{C_{D} \rho S}}$. Taking $\rho=1, S=1$, and $m \mathrm{~g}=(0,0,-1)$ to construct a unit model problem, one can solve for the velocity as a function of position for an object initially at rest and falling in the $-z$ direction as

$$
u_{z}(z)=\sqrt{4\left(1-e^{0.5 z}\right)}
$$

Figure A.3 plots velocity as a function of distance for the theoretical result and numerical experiments run with $\Delta t=0.1,0.2$ and 0.4 . As expected halving the timestep halves the maximum error in the simulations providing the expected order of accuracy. All simulations converge to the correct terminal velocity $\left(u_{\infty}=-2.0\right.$ ) since the external force becomes a constant at this limit.

Smart [1] presents an exact solution to Euler's equations of motion that corresponds to a tumbling and spinning cylinder. For this example there is no translation, and in the current notation the inertial properties are given by

$$
\begin{aligned}
I_{1} & =I_{2} \\
I_{1}-I_{3} & =I_{2}-I_{3}=\alpha I_{1}
\end{aligned}
$$




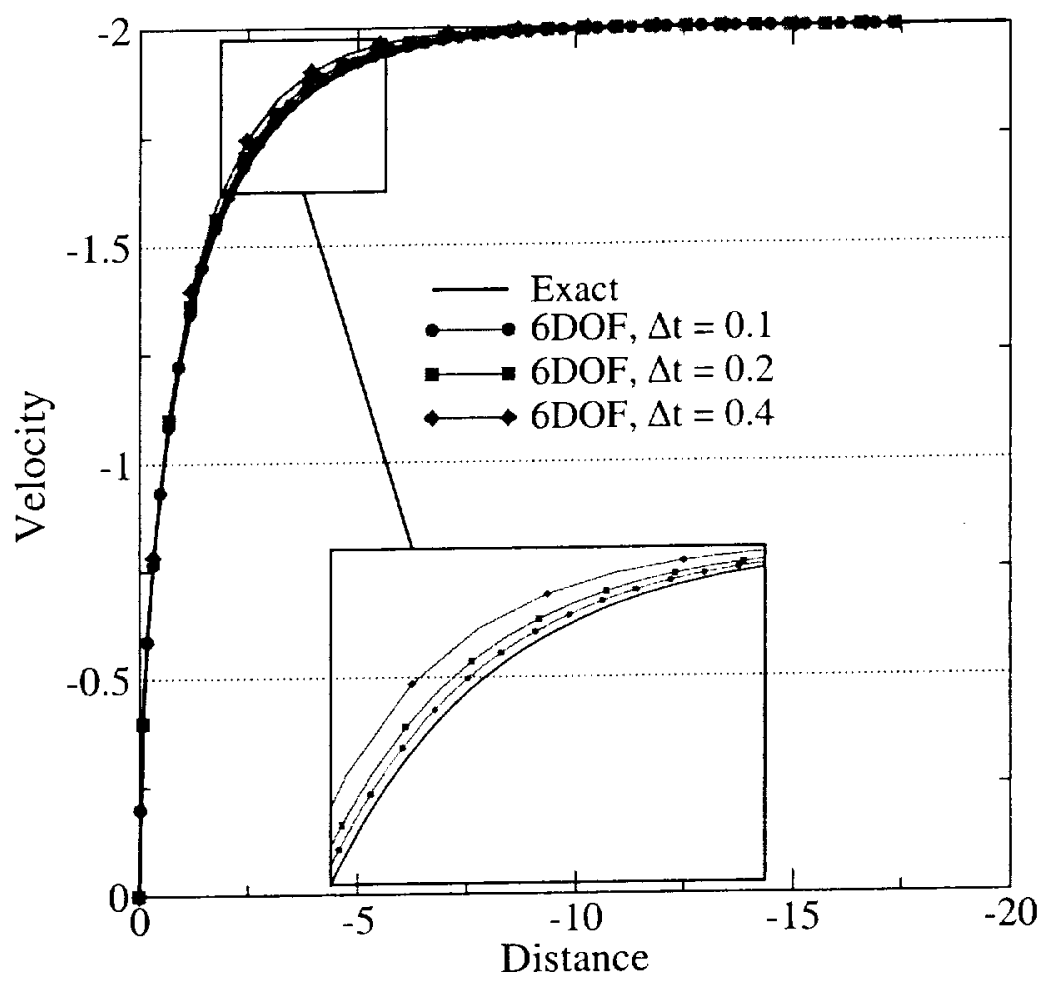

Figure A.3: Velocity vs distance evolution of a sphere falling in a gravitational field subject to air resistance.

The analytic solution of the time evolution of the angular velocities is

$$
\begin{aligned}
& \omega_{1}=a \cos (\lambda t) \\
& \omega_{2}=b \sin (\lambda t) \\
& \omega_{3}=c
\end{aligned}
$$

Setting $I_{1}=I_{2}=1.0, \alpha=0.5, a=1.0$ and $c=0.5$ gives $I_{3}=0.5$ and $\lambda=0.25$.

At $t=0$, the 6 -DOF model is initialized with $\boldsymbol{\omega}=(1.0,0.0,0.5)$. Figure A.4 shows the system's response to this initial condition compared against the analytic solution with $b=-1$. Evolution of the numerically-integrated angular velocities are plotted for $\Delta t=1.0,2.0$ and 4.0. Since the integration is formally fourth order, the results converge very quickly and only the symbols for $\Delta t=4.0$ clearly differ from the theoretical curve. With a $\Delta t$ of 1.0 there are approximately 12 samples per wavelength. Table A.1 provides a quantitative comparison of the convergence, listing the error in $\omega_{1}$ at $t=100$ as $\Delta t$ increases. Since the error increases with time, this is the maximum error or the interval $t=[0,100]$. The data in Table A.1 shows fourth order asymptotic convergence, as expected. 


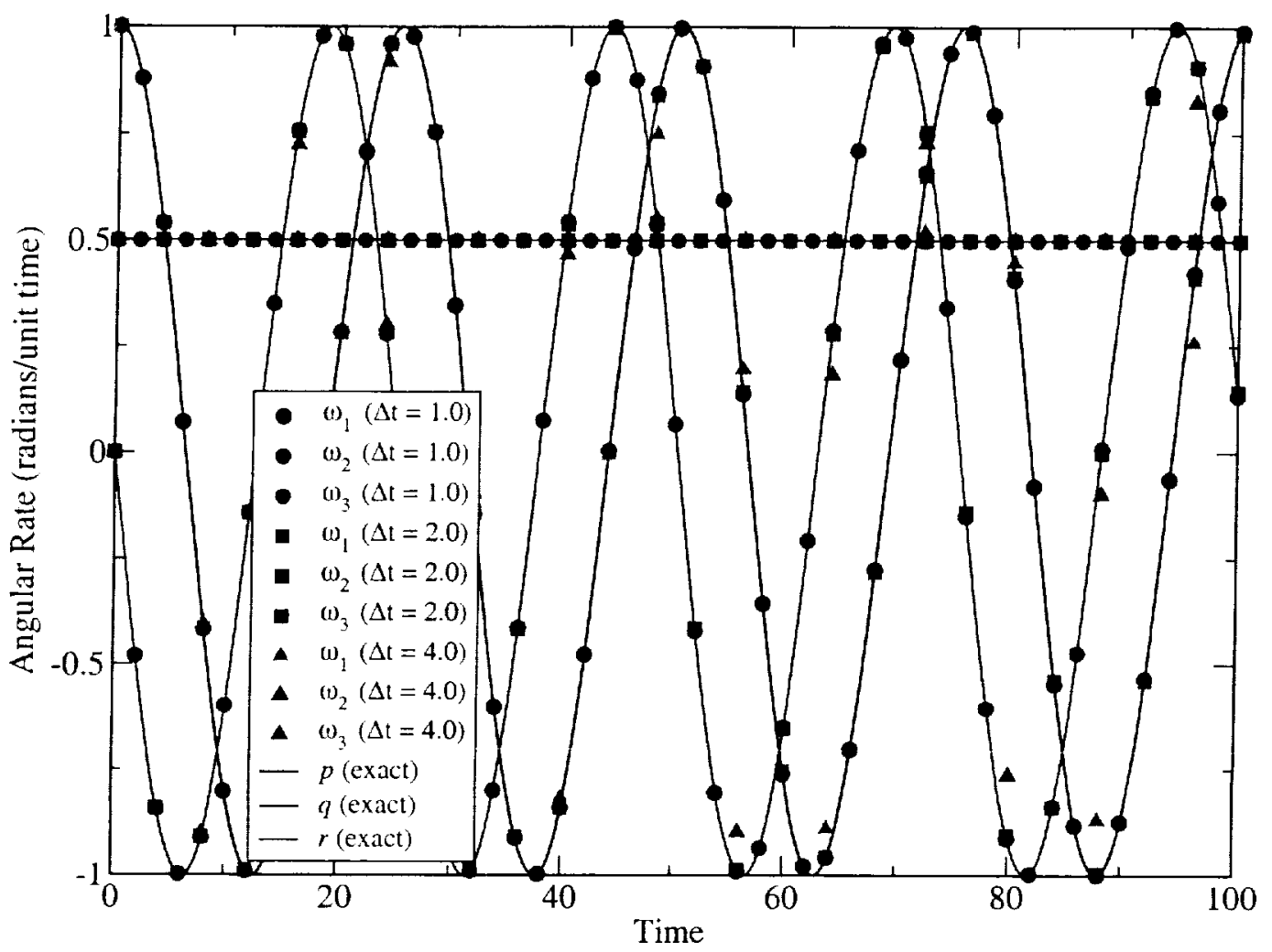

Figure A.4: Time evolution of numerical integration for angular velocities compared with an exact solution of Euler's laws of motion from Smart [1].

\begin{tabular}{||c||c|c|c||}
\hline$\Delta t$ & $\begin{array}{c}\text { \%Error in } \omega_{1} \\
\text { at } t=100\end{array}$ & Improvement & Order of accuracy \\
\hline \hline 0.25 & $5.89 \mathrm{E}-5$ & 20.96 & 4.39 \\
\hline 0.50 & $1.2047 \mathrm{E}-3$ & 22.80 & 4.51 \\
\hline 1.0 & 0.02747 & 25.08 & 4.65 \\
\hline 2.0 & 0.6890 & 24.09 & 4.59 \\
\hline 4.0 & 16.60 & - & - \\
\hline
\end{tabular}

Table A.1: Accuracy of numerical integration of Euler's Eqs. of motion for coupled rotation of a cylinder.

While the system of Euler's Eqns. decouples when the rotation axes are aligned with any one of the principal axes of a body, stability analysis shows that this rotation is only stable around the minor or major axis - rotation around the semi-major axis is unstable. The coupling of the system means that any small perturbation about the semi-major axis will excite rotation about the others (cf. Thompson[2]). With $I_{1}=1, I_{2}=10$, and $I_{3}=100$, the 6 -DOF model was initialized with $\boldsymbol{\omega}=(0,0,1)$, 
prescribing rotation around the major axis. The system was perturbed by imposing a moment with magnitude 0.01 about the minor axis over the first time step $(\Delta t=0.1)$. Figure A.5 shows the system response in terms of the angular rates around the minor and semi-major axis. As expected, the initial perturbation excites oscillations around both of these axes, but these oscillations disappear rapidly as the system stabilizes. Since the system is lossless (i.e. contains no physical dissipation), the rotational energy of the system must be conserved. Figure A.6 shows the Euler angles of the object, revealing that the initial oscillations are transformed into a steady, but extremely small, oscillation about both the minor and semi-major axes. Figure A.7 shows that this oscillation persists undamped, as expected from a lossless system.

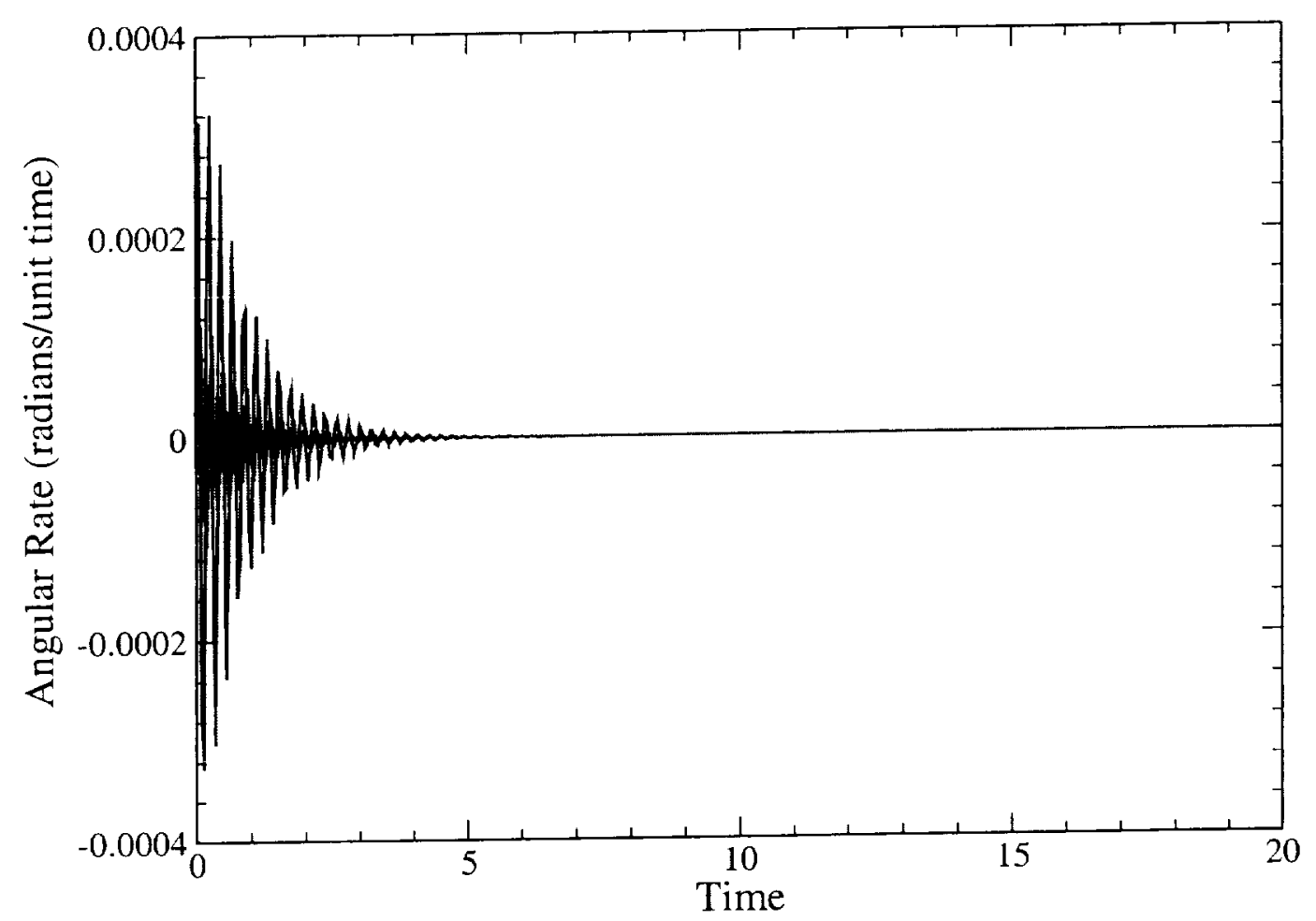

Figure A.5: Time evolution of angular velocity around minor (red) and semi-major (blue) axes for a system spinning around major axis. System is perturbed at $t=0$ with an impulsive couple around the minor axis.

Contrast the results of Figs. A.5-A.7 with those shown in Fig. A.8. In the example shown in Fig. A.8, the initial angular velocity is prescribed as $\omega=(0,1,0)$ and the moments of inertia are unchanged from the previous example. Spin is therefore around the semi-major axis. When the same initial perturbation is applied, the perturbation is amplified, resulting in spin around all three axes. It's clear from the plot that the magnitude of the resulting angular rates is proportional to the moments of inertia 


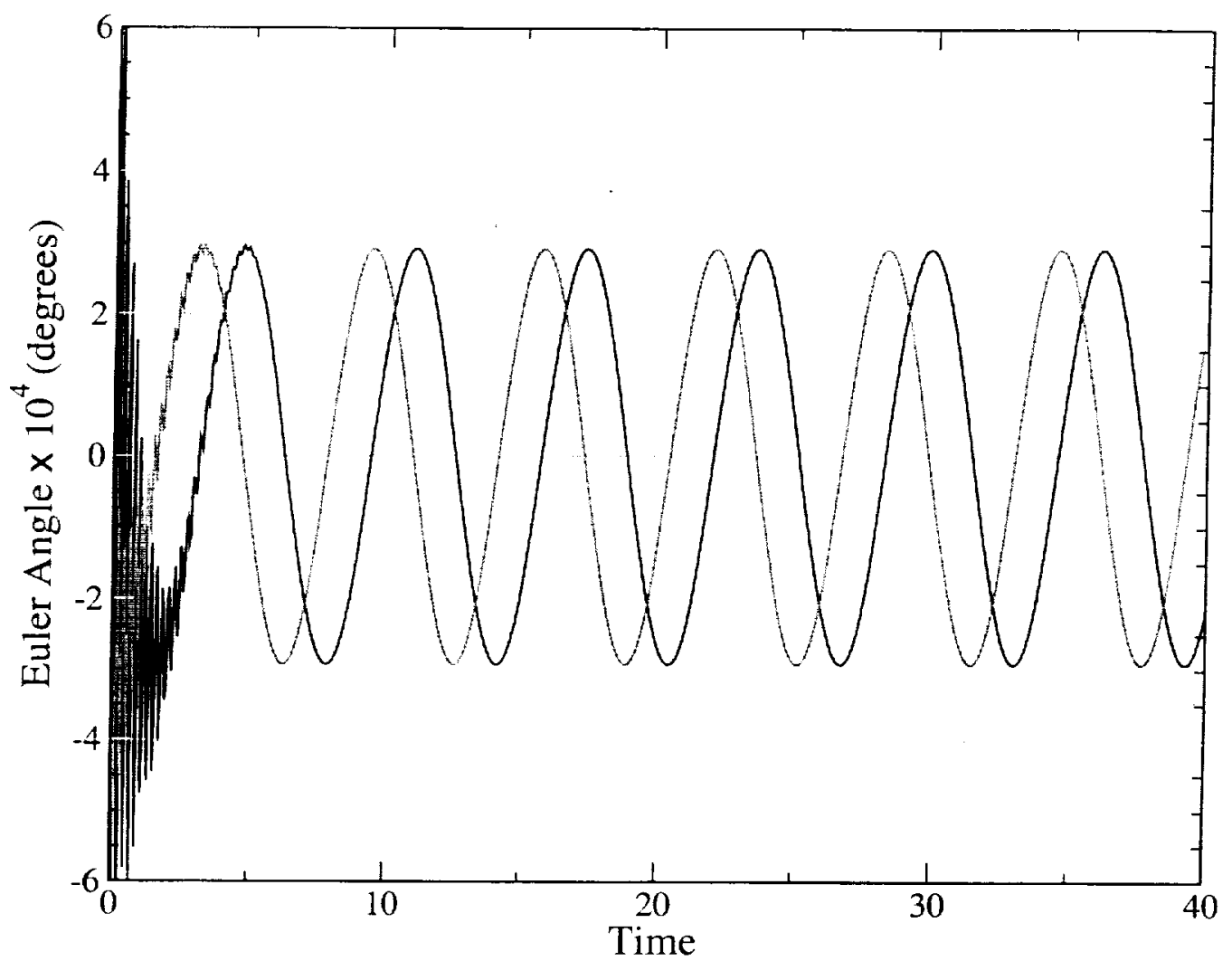

Figure A.6: Time evolution of Euler angles showing small oscillation excited by perturbation of system spinning around major axis. System is perturbed at $t=0$ with an impulsive couple around the minor axis.

around these axes. Again, since the system is lossless, this tumbling behavior persists undamped.

\section{References}

[1] Smart, Howard, Advanced Dynamics.

[2] Thompson, W.T., Introduction to Spacecraft Dynamics. John Wiley \& Sons, 1963. 


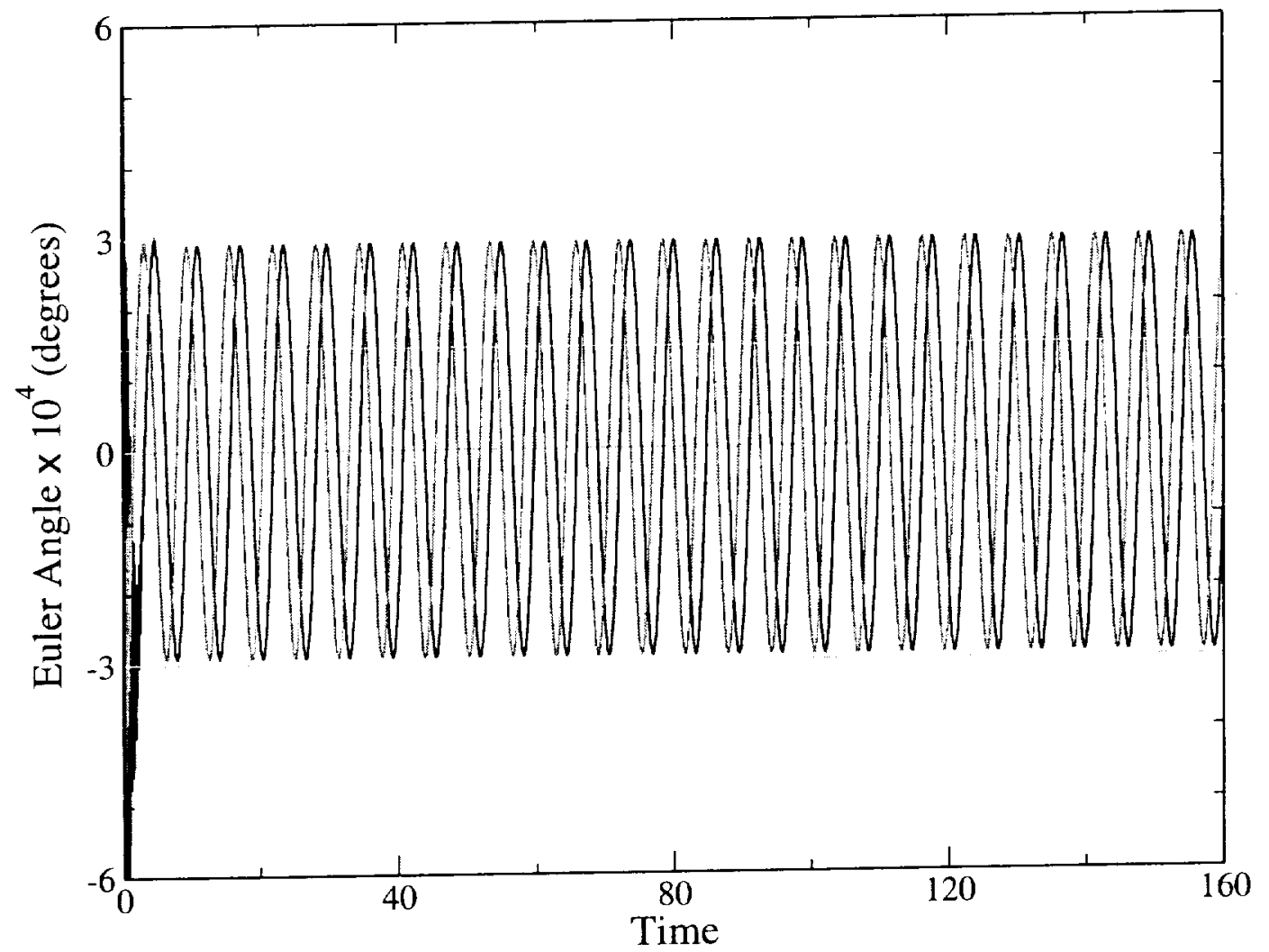

Figure A.7: Late time response of system in Fig. A.6, showing undamped response. System is perturbed at $t=0$ with an impulsive couple around the minor axis. 


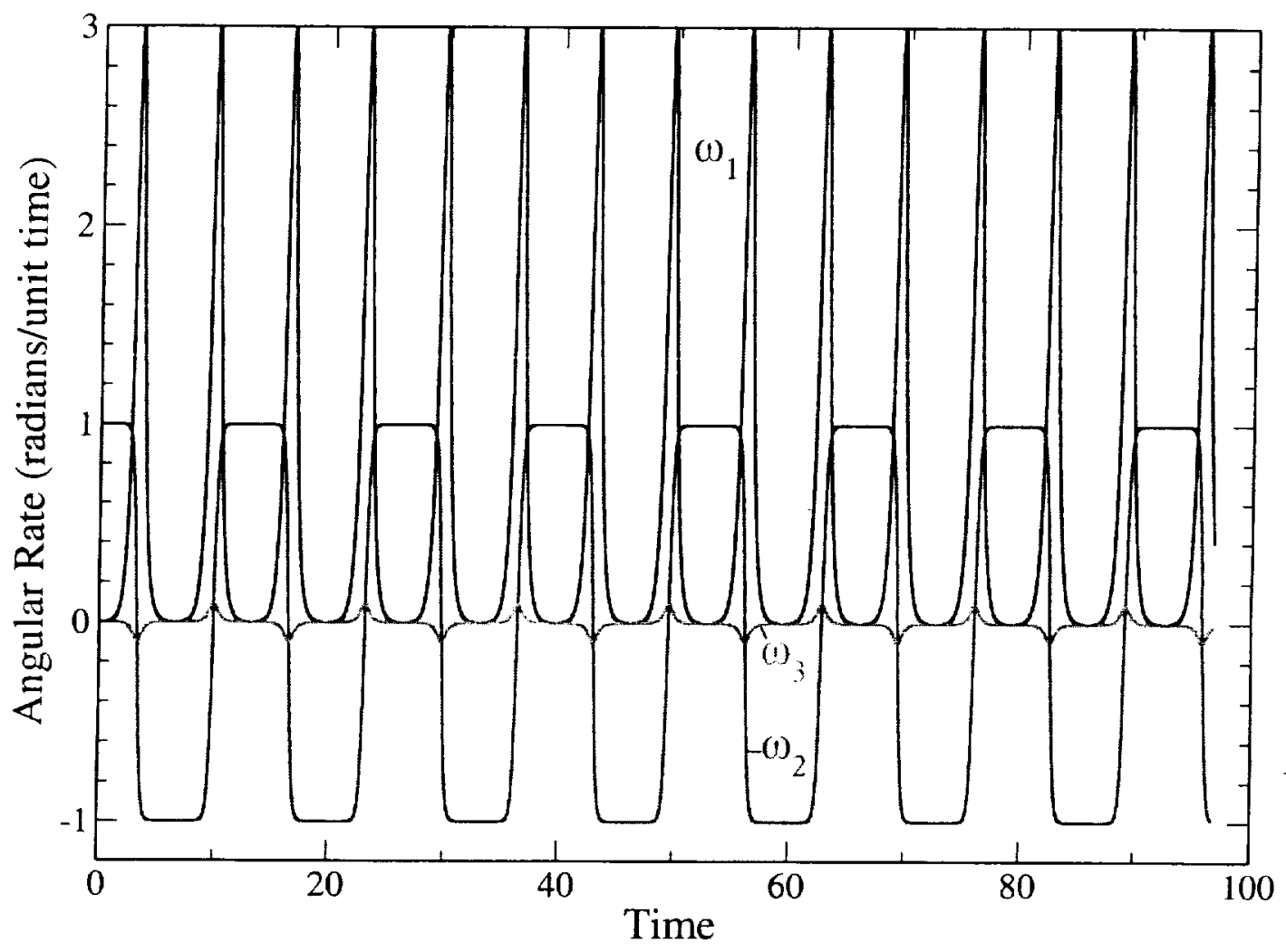

Figure A.8: Angular rate response to an initial perturbation for an object initially spinning around its semi-major axis at rate $\omega_{2}=1$. Coupling quickly leads to a tumbling motion. 